\title{
A risk attitudinal ranking method for interval-valued intuitionistic fuzzy numbers based on novel attitudinal expected score and accuracy functions
}

\author{
Jian $\mathrm{Wu}^{\mathrm{a}, \mathrm{b}}$, Francisco Chiclana ${ }^{\mathrm{b}}$ \\ ${ }^{a}$ School of Economics and Management, Zhejiang Normal University, Jinhua, Zhejiang, China \\ ${ }^{b}$ Centre for Computational Intelligence, Faculty of Technology, De Montfort University, Leicester, UK
}

\begin{abstract}
This article investigates new score and accuracy functions for ranking interval-valued intuitionistic fuzzy numbers (IVIFNs). The novelty of these functions is that they allow the comparison of IVIFNs by taking into account of the decision makers' attitudinal character. The new attitudinal expected score and accuracy functions extend $\mathrm{Xu}$ and Chen's score and accuracy degree functions, and verify the following set of properties: (1) boundedness; (2) monotonicity; (3) commutativity; and (4) symmetry. These novel functions are used to propose a total order on the set of IVIFNs, and to develop an interval-valued intuitionistic fuzzy multi-attribute decision making selection process in which the final result depends on the decision maker's risk attitude. In addition, a ranking sensitivity analysis with respect to the risk attitude is provided.
\end{abstract}

Keywords: Multi-attribute decision-making, interval-valued intuitionistic sets, attitudinal expected score function, attitudinal expected accuracy function, COWA operator

\section{Introduction}

Atanassov [1] introduced the concept of intuitionistic fuzzy sets (IFSs), which is characterised by both the membership and non-membership functions and therefore it generalises the concept of fuzzy set [2,3]. Subsequently, Atanassov and Gargov [4] extended IFSs with the introduction of the concept of interval-valued intuitionistic fuzzy sets (IVIFSs). The notions of IFSs and IVIFSs are interesting and very useful in modelling real life problems with imprecision or uncertainty and they have been applied to many

Email addresses: jyajian@163.com (Jian Wu), chiclana@dmu.ac.uk (Francisco Chiclana) 
different fields, including multiple attribute decision making (MADM) [5-9], group decision making (GDM) [10-12], supplier selection [13, 14], robot selection [15] and artificial intelligence [16].

The first step of any MADM process with information modelled using IFSs is to fuse the intuitionistic fuzzy assessment values of the different attributes into a collective intuitionistic fuzzy assessment via an appropriate aggregation operator [17]. Once this step has been completed, the aggregated intuitionistic fuzzy numbers are compared to produce a final ranking of the alternatives. Consequently, an active research topic is the investigation of intuitionistic fuzzy MADM that includes suitable and valid intuitionistic fuzzy aggregation operators. Since Xu [18] developed the intuitionistic fuzzy ordered weighted averaging (IFOWA) operator, extensive research work has been carried out to develop aggregation operators for both IFSs and/or IVIFSs (see for example [19-21]). However, the above cited intuitionistic fuzzy operators are based on additive measures and are not suitable to aggregate inter-dependent criteria. To resolve this issue, Tan and Chen $[22,23]$ proposed the intuitionistic fuzzy Choquet integral operator and the generalized interval-valued intuitionistic fuzzy geometric aggregation operator for multiattribute interval-valued intuitionistic fuzzy group decision making problems.

Another active research topic regards the development of score degree and accuracy degree functions to make possible the comparison of criterion values that are expressed by IFSs and IVIFSs, respectively. A comprehensive comparative analysis of existing score degree and accuracy degree functions to date can be found in [24]. Chen and Tan in [25] developed a score degree function for IFSs based on the membership degree and nonmembership degree functions, which was later improved by Hong and Choi in [26] with the addition of an accuracy degree function. In addition, Hong and Choi argued about the similarity between the role of the score degree and the accuracy degree functions of IFSs and that of the mean and the variance in statistics. Subsequently, other improved score degree and accuracy degree functions have been proposed in [27-32]. Also these functions are extended to the cases of triangular intuitionistic fuzzy numbers $[33,34]$ and intuitionistic linguistic numbers [35-37]. It is worth mentioning the score degree and accuracy degree functions recently developed by $\mathrm{Xu}$ and Chen in [38] to propose an order relation on the set of interval-valued intuitionistic fuzzy numbers (IVIFNs). However, as it will be proved later with a counter-example (Example 1), the order relation derived 
from the application of $\mathrm{Xu}$ and Chen's score degree and accuracy degree functions is not total. In a attempt to resolve this drawback, Ye in [39] and Lakshmana Gomathi Nayagam in [40] proposed alternative accuracy degree functions, respectively, which they claimed produced a total ordering of IVIFNs. However, these alternative degree functions are not superior to the existing accuracy degree functions but equivalent in the course of comparing any two interval-valued intuitionistic fuzzy numbers'. As it will be shown later in Section 3.3, these score degree and accuracy degree functions do not capture well all the information contained in IVIFNs and consequently can lead to a lack of precision in the final ordering of IVIFNs. We believe that this is because these functions are simple and straight forward extensions of their respective proposals for the case of IFNs. An important limitation of the above approaches resides in the fact that they do not take into account the attitudinal character of decision makers. Yager in [41] pointed out that the attitudinal character of each decision maker may affect the final ranking order of fuzzy numbers (FNs). The problem of ordering FNs, though, has been extensively studied and an agreed conclusion is that there is no unique best approach to do this. Recall that FNs are particular cases of IVIFNs. Thus, the same conclusion applies to IVIFNs. Therefore, it is important to develop a methodology that best captures the decision maker's risk attitude regarding the ranking IVIFNs.

In order to achieve this, the the remainder of this paper is organised as follows: The next section briefly reviews the main score degree and accuracy degree functions of IVIFSs and an analysis of their relationships as well as their associated drawbacks is carried out, which it is used in Section 3 to support the development of novel attitudinal expected score and accuracy functions of IVIFNs driven by the decision maker's attitudinal character. In this section, it is also proved that the new attitudinal expected functions extend $\mathrm{Xu}$ and Chen's score degree and accuracy degree functions. The following desirable properties are proved to be satisfied by the new attitudinal expected functions: (i) boundedness; (ii) monotonicity; (iii) commutativity; and (iv) symmetry. This section is completed with the definition of a total order relation on the set of IVIFNs. Section 4 presents a sensitivity analysis with respect to the attitudinal character and a resolution process of MADM problems in an interval-valued intuitionistic fuzzy environment. Finally, in Section 5 conclusions are drawn and suggestions made for further work. 


\section{Preliminaries}

This section presents the key concepts related to IVIFSs that will be used throughout this paper. First, we present Atanassov and Gargov's defintion of the notion of IVIFS, which is characterised by a membership function and a non-membership function that take interval numbers rather than crisp numbers, as introduced in [4].

Definition 1 (Interval-Valued IFS (IVIFS)). Let $I N T([0,1])$ be the set of all closed subintervals of the unit interval and $X$ be a universe of discourse. An interval-valued IFS (IVIFS) $A$ over $X$ is given as:

$$
A=\left\{\left\langle x, \widetilde{\mu}_{A}(x), \widetilde{\nu}_{A}(x)\right\rangle \mid x \in X\right\}
$$

where $\widetilde{\mu}_{A}(x), \widetilde{\nu}_{A}(x) \in \operatorname{INT}([0,1])$, represent the membership and the non-membership degrees of the element $x$ to the set $A$ subject to the following constraint

$$
0 \leq \sup \widetilde{\mu}_{A}(x)+\sup \widetilde{\nu}_{A}(x) \leq 1, \forall x \in X
$$

Denoting by $\widetilde{\mu}_{A L}(x), \widetilde{\mu}_{A U}(x), \widetilde{\nu}_{A L}(x)$ and $\widetilde{\nu}_{A U}(x)$ the lower and upper end points of $\widetilde{\mu}_{A}(x)$ and $\widetilde{\nu}_{A}(x)$, respectively, an IVIFS can be represented as

$$
\begin{aligned}
& A=\left\{\left\langle x,\left[\widetilde{\mu}_{A L}(x), \widetilde{\mu}_{A U}(x)\right],\left[\widetilde{\nu}_{A L}(x), \widetilde{\nu}_{A U}(x)\right]\right\rangle \mid\right. \\
& \left.\quad x \in X: 0 \leq \widetilde{\mu}_{A U}(x)+\widetilde{\nu}_{A U}(x) \leq 1, \widetilde{\mu}_{A L}(x) \wedge \widetilde{\nu}_{A L}(x) \geq 0\right\}
\end{aligned}
$$

Recall that given two IVIFSs, $A$ and $B$, Atanassov and Gargov containment concept is modelled as follows [4]:

$$
A \subseteq B
$$

iff

$\forall x \in X: \widetilde{\mu}_{A L}(x) \leq \widetilde{\mu}_{B L}(x) \wedge \widetilde{\mu}_{A U}(x) \leq \widetilde{\mu}_{B U}(x) \wedge \widetilde{\nu}_{A L}(x) \geq \widetilde{\nu}_{B L}(x) \wedge \widetilde{\nu}_{A U}(x) \geq \widetilde{\nu}_{B U}(x)$

The hesitancy degree function of an IVIFS is:

$$
\widetilde{\pi}_{A}(x)=\left[1-\widetilde{\mu}_{A U}(x)-\widetilde{\nu}_{A U}(x), 1-\widetilde{\mu}_{A L}(x)-\widetilde{\nu}_{A L}(x)\right] .
$$

Given $x \in X$,

$$
\left(\left[\widetilde{\mu}_{A L}(x), \widetilde{\mu}_{A U}(x)\right],\left[\widetilde{\nu}_{A L}(x), \widetilde{\nu}_{A U}(x)\right]\right)
$$


will be referred to as an interval-valued intuitionistic fuzzy number (IVIFN). For convenience, an IVIFN will be denoted by $\left(\left[\mu^{-}, \mu^{+}\right],\left[\nu^{-}, \nu^{+}\right]\right)$.

Given two IVIFNs $\widetilde{\alpha}_{1}=\left(\left[\mu_{1}^{-}, \mu_{1}^{+}\right],\left[\nu_{1}^{-}, \nu_{1}^{+}\right]\right)$and $\widetilde{\alpha}_{2}=\left(\left[\mu_{2}^{-}, \mu_{2}^{+}\right],\left[\nu_{2}^{-}, \nu_{2}^{+}\right]\right)$, we have the following definition of containment [4]:

$$
\widetilde{\alpha}_{1} \subseteq \widetilde{\alpha}_{2} \text { iff } \mu_{1}^{-} \leq \mu_{2}^{-}, \mu_{1}^{+} \leq \mu_{2}^{+}, \nu_{1}^{-} \geq \nu_{2}^{-} \text {, and } \nu_{1}^{+} \geq \nu_{2}^{+}
$$

Also, the main arithmetic operations can be expressed in terms of the interval lower and upper bounds as follows [1, 42]:

1) $\overline{\widetilde{\alpha}}_{1}=\left(\left[\nu_{1}^{-}, \nu_{1}^{+}\right],\left[\mu_{1}^{-}, \mu_{1}^{+}\right]\right)$

2) $\widetilde{\alpha}_{1} \oplus \widetilde{\alpha}_{2}=\left(\left[\mu_{1}^{-}+\mu_{2}^{-}-\mu_{1}^{-} \cdot \mu_{2}^{-}, \mu_{1}^{+}+\mu_{2}^{+}-\mu_{1}^{+} \cdot \mu_{2}^{+}\right],\left[\nu_{1}^{-} \cdot \nu_{2}^{-}, \nu_{1}^{+} \cdot \nu_{2}^{+}\right]\right)$

3) $\widetilde{\alpha}_{1} \otimes \widetilde{\alpha}_{2}=\left(\left[\mu_{1}^{-} \cdot \mu_{2}^{-}, \mu_{1}^{+} \cdot \mu_{2}^{+}\right],\left[\nu_{1}^{-}+\nu_{2}^{-}-\nu_{1}^{-} \cdot \nu_{2}^{-}, \nu_{1}^{+}+\nu_{2}^{+}-\nu_{1}^{+} \cdot \nu_{2}^{+}\right]\right)$

4) $\lambda \cdot \widetilde{\alpha}_{1}=\left(\left[1-\left(1-\mu_{1}^{-}\right)^{\lambda}, 1-\left(1-\mu_{1}^{+}\right)^{\lambda}\right],\left[\left(\nu_{1}^{-}\right)^{\lambda},\left(\nu_{1}^{+}\right)^{\lambda}\right]\right)$

5) $\widetilde{\alpha}_{1}^{\lambda}=\left(\left[\left(\mu_{1}^{-}\right)^{\lambda},\left(\mu_{1}^{+}\right)^{\lambda}\right],\left[1-\left(1-\nu_{1}^{-}\right)^{\lambda}, 1-\left(1-\nu_{1}^{+}\right)^{\lambda}\right]\right)$

The score degree and accuracy degree functions play a key role in MADM problems with interval-valued intuitionistic fuzzy information because they allow the comparison of criteria values using IVIFNs. For IVIFNs, Xu and Chen proposed in [38] the following score degree and accuracy degree functions:

Definition 2 (Xu and Chen's IVIFN Score and Accuracy Functions [38]). Let $\widetilde{\alpha}=$ $\left(\left[\mu^{-}, \mu^{+}\right],\left[\nu^{-}, \nu^{+}\right]\right)$be an IVIFN. The score degree and accuracy degree functions of $\widetilde{\alpha}$ are represented, respectively, by

$$
\widetilde{S}_{X C}(\widetilde{\alpha})=\frac{\mu^{-}+\mu^{+}-\nu^{-}-\nu^{+}}{2}
$$

and

$$
\widetilde{A}_{X C}(\widetilde{\alpha})=\frac{\mu^{-}+\mu^{+}+\nu^{-}+\nu^{+}}{2}
$$

Notice that $S_{X C}(\widetilde{\alpha}) \in[-1,1]$, while $A_{X C}(\widetilde{\alpha}) \in[0,1]$, and that both functions are related as follows:

$$
A_{X C}(\widetilde{\alpha})=S_{X C}(\widetilde{\alpha})+\left(\nu^{-}+\nu^{+}\right) .
$$

Thus, the following result is proved: 
Proposition 1. Given an IVIFN $\widetilde{\alpha}=\left(\left[\mu^{-}, \mu^{+}\right],\left[\nu^{-}, \nu^{+}\right]\right)$, the following inequality holds:

$$
\widetilde{S}_{X C}(\widetilde{\alpha}) \leq \widetilde{A}_{X C}(\widetilde{\alpha})
$$

The score degree function is monotonic increasing with respect to the containment relation of IVIFNs are the following result proves:

Proposition 2. Given two IVIFNs $\widetilde{\alpha}_{1}$ and $\widetilde{\alpha}_{2}$ such that $\widetilde{\alpha}_{1} \subseteq \widetilde{\alpha}_{2}$ then it is $\widetilde{S}_{X C}\left(\widetilde{\alpha}_{1}\right) \leq$ $\widetilde{S}_{X C}\left(\widetilde{\alpha}_{2}\right)$.

Proof. Recall that $\widetilde{\alpha}_{1} \subseteq \widetilde{\alpha}_{2}$ is equivalent to $\mu_{1}^{-} \leq \mu_{2}^{-}, \mu_{1}^{+} \leq \mu_{2}^{+}, \nu_{1}^{-} \geq \nu_{2}^{-}, \nu_{1}^{+} \geq \nu_{2}^{+}$. Therefore it is

$$
\mu_{1}^{-}+\mu_{1}^{+}-\nu_{1}^{-}-\nu_{1}^{+} \leq \mu_{2}^{-}+\mu_{2}^{+}-\nu_{2}^{-}-\nu_{2}^{+}
$$

The score degree and accuracy degree are used by $\mathrm{Xu}$ and Chen to propose the following IVIFNs two level ranking method:

Definition 3 (Xu amnd Chen's IVIFNs Order Relation [38]). Given two IVIFNs $\widetilde{\alpha}_{1}=\left(\left[\mu_{1}^{-}, \mu_{1}^{+}\right],\left[\nu_{1}^{-}, \nu_{1}^{+}\right]\right)$and $\widetilde{\alpha}_{2}=\left(\left[\mu_{2}^{-}, \mu_{2}^{+}\right],\left[\nu_{2}^{-}, \nu_{2}^{+}\right]\right)$, the following ordering relation can be established:

(1) If $\widetilde{S}_{X C}\left(\widetilde{\alpha}_{1}\right)<\widetilde{S}_{X C}\left(\widetilde{\alpha}_{2}\right)$ then $\widetilde{\alpha}_{1}<\widetilde{\alpha}_{2}$.

(2) If $\widetilde{S}_{X C}\left(\widetilde{\alpha}_{1}\right)=\widetilde{S}_{X C}\left(\widetilde{\alpha}_{2}\right)$ :

(i) If $\widetilde{A}_{X C}\left(\widetilde{\alpha}_{1}\right)<\widetilde{A}_{X C}\left(\widetilde{\alpha}_{2}\right)$ then $\widetilde{\alpha}_{1}<\widetilde{\alpha}_{2}$.

(ii) If $\widetilde{A}_{X C}\left(\widetilde{\alpha}_{1}\right)=\widetilde{A}_{X C}\left(\widetilde{\alpha}_{2}\right)$ then $\widetilde{\alpha}_{1}=\widetilde{\alpha}_{2}$.

In the first level, the score degree is used to rank the IVIFNs, and the second level is only applied when two IVIFNs have same score degrees, in which case their accuracy degrees are used to discern the order relation between the IVIFSs. Two IVIFSs are considered equivalent in term of ordering when both have same score degree and accuracy degree.

Ye in [39] proposed an alternative expression for the accuracy degree function of an IVIFN to Xu and Chen's accuarcy degree function, but with same range of values, $[-1,1]$ : 
Definition 4 (Ye's IVIFN Accuracy Function [39]). The accuracy degree function of an IVIFN $\widetilde{\alpha}=\left(\left[\mu^{-}, \mu^{+}\right],\left[\nu^{-}, \nu^{+}\right]\right)$can be represented by

$$
\widetilde{A}_{Y}(\widetilde{\alpha})=\mu^{-}+\mu^{+}+\frac{\nu^{-}+\nu^{+}}{2}-1
$$

Notice that in the above definitions, the hesitancy degree function of IVIFNs is not implemented, which is not the case in the following accuracy degree function introduced by Lakshmana and Sivaraman in [40]:

Definition 5 (Lakshmana and Sivaraman's IVIFN Accuracy Function [40]). The hesitancy based accuracy degree function of an IVIFN $\widetilde{\alpha}=\left(\left[\mu^{-}, \mu^{+}\right],\left[\nu^{-}, \nu^{+}\right]\right)$can be represented by

$$
\widetilde{A}_{L S_{\delta}}(\widetilde{\alpha})=\frac{\text { membership degree }+\delta \cdot(\text { hesitancy degree })}{2}
$$

i.e.

$$
\widetilde{A}_{L S_{\delta}}(\widetilde{\alpha})=\frac{\mu^{-}+\mu^{+}+\delta \cdot\left(2-\mu^{-}-\mu^{+}-\nu^{-}-\nu^{+}\right)}{2}
$$

where $\delta \in[0,1]$ is a parameter that is used to control the individual's intentions as follows: $\delta=1$ corresponds to an optimistic individual, while a pessimistic one would have a value of $\delta=0$.

An alternative expression for $\widetilde{A}_{L S_{\delta}}(\widetilde{\alpha})$ is:

$$
\widetilde{A}_{L S_{\delta}}(\widetilde{\alpha})=\frac{1-\delta}{2} \cdot\left(\mu^{-}+\mu^{+}\right)-\frac{\delta}{2} \cdot\left(\nu^{-}+\nu^{+}\right)+\delta .
$$

Using expression (10), it is easy to prove that Lakshmana and Sivaraman's accuracy function is monotonic increasing with respect to the containment relation of IVIFNs:

Proposition 3. Given two IVIFNs $\widetilde{\alpha}_{1}$ and $\widetilde{\alpha}_{2}$ such that $\widetilde{\alpha}_{1} \subseteq \widetilde{\alpha}_{2}$, then it is $\widetilde{A}_{L S_{\delta}}\left(\widetilde{\alpha}_{1}\right) \leq$ $\widetilde{A}_{L S_{\delta}}\left(\widetilde{\alpha}_{2}\right)$.

Notice that $\widetilde{A}_{Y}(\widetilde{\alpha})$ and $\widetilde{A}_{L S_{\delta}}(\widetilde{\alpha})$ can be re-written as follows:

$$
\begin{gathered}
\widetilde{A}_{Y}(\widetilde{\alpha})=\widetilde{S}_{X C}(\widetilde{\alpha})+\frac{3}{2} \cdot \widetilde{A}_{X C}(\widetilde{\alpha})-1 \\
\widetilde{A}_{L S_{\delta}}\left(\widetilde{\alpha}_{1}\right)=\frac{1}{2} \cdot \widetilde{S}_{X C}(\widetilde{\alpha})+\frac{1-2 \cdot \delta}{2} \cdot \widetilde{A}_{X C}(\widetilde{\alpha})+\delta .
\end{gathered}
$$

The following relationships between the above score degree and accuracy degree functions are established: 
Proposition 4. The IVIFN accuracy degree function $\widetilde{A}_{L S_{\delta}}$ and the score degree function $S_{X C}$ are related as follows:

$$
\widetilde{A}_{L S_{0.5}}(\widetilde{\alpha})=\frac{1}{2} \cdot \widetilde{S}_{X C}(\widetilde{\alpha})+\frac{1}{2}
$$

and therefore are equivalent in the ordering IVIFNs.

Proposition 5. Given any two IVIFNs $\widetilde{\alpha}_{1}=\left(\left[\mu_{1}^{-}, \mu_{1}^{+}\right],\left[\nu_{1}^{-}, \nu_{1}^{+}\right]\right)$and $\widetilde{\alpha}_{2}=\left(\left[\mu_{2}^{-}, \mu_{2}^{+}\right],\left[\nu_{2}^{-}, \nu_{2}^{+}\right]\right)$, we have:

1. If $\widetilde{S}_{X C}\left(\widetilde{\alpha}_{1}\right)=\widetilde{S}_{X C}\left(\widetilde{\alpha}_{2}\right)$ and $\widetilde{A}_{X C}\left(\widetilde{\alpha}_{1}\right)=\widetilde{A}_{X C}\left(\widetilde{\alpha}_{2}\right)$ then

1.a) $\widetilde{A}_{Y}\left(\widetilde{\alpha}_{1}\right)=\widetilde{A}_{Y}\left(\widetilde{\alpha}_{2}\right)$.

1.b) $\widetilde{A}_{L S_{\delta}}\left(\widetilde{\alpha}_{1}\right)=\widetilde{A}_{L S_{\delta}}\left(\widetilde{\alpha}_{2}\right)$.

2. If $\widetilde{S}_{X C}\left(\widetilde{\alpha}_{1}\right)=\widetilde{S}_{X C}\left(\widetilde{\alpha}_{2}\right)$ and $\widetilde{A}_{X C}\left(\widetilde{\alpha}_{1}\right)<\widetilde{A}_{X C}\left(\widetilde{\alpha}_{2}\right)$ then

2.a) $\widetilde{A}_{Y}\left(\widetilde{\alpha}_{1}\right)<\widetilde{A}_{Y}\left(\widetilde{\alpha}_{2}\right)$.

2.b1) $\widetilde{A}_{L S_{\delta}}\left(\widetilde{\alpha}_{1}\right)<\widetilde{A}_{L S_{\delta}}\left(\widetilde{\alpha}_{2}\right)$ when $\delta<0.5$.

2.b2) $\widetilde{A}_{L S_{\delta}}\left(\widetilde{\alpha}_{1}\right)=\widetilde{A}_{L S_{\delta}}\left(\widetilde{\alpha}_{2}\right)$ when $\delta=0.5$.

2.b3) $\widetilde{A}_{L S_{\delta}}\left(\widetilde{\alpha}_{1}\right)>\widetilde{A}_{L S_{\delta}}\left(\widetilde{\alpha}_{2}\right)$ when $\delta>0.5$.

As mentioned before, the score degree and accuracy degree functions are widely used in interval-valued intuitionistic fuzzy MADM problems to produce a final ranking of alternatives. However, as the following example illustrates, they are unable to discriminate between all pairs of IVIFNs in terms of ranking:

Example 1. The following two IVIFNs

$$
\widetilde{\alpha}_{1}=([0.15,0.35],[0.2,0.4]) \text { and } \widetilde{\alpha}_{2}=([0.2,0.3],[0.2,0.35])
$$

have the same score value $\widetilde{S}_{X C}\left(\widetilde{\alpha}_{1}\right)=\widetilde{S}_{X C}\left(\widetilde{\alpha}_{2}\right)=-0.05$, and therefore the accuracy value is to be used to rank them. However, in this case we have $\widetilde{A}_{X C}\left(\widetilde{\alpha}_{1}\right)=\widetilde{A}_{X C}\left(\widetilde{\alpha}_{2}\right)=0.55$. From expressions $(7)$ and (9), and in accordance to Proposition 5 , we have $\widetilde{A}_{Y}\left(\widetilde{\alpha}_{1}\right)=$ $\widetilde{A}_{Y}\left(\widetilde{\alpha}_{1}\right)=-0.025$ and $\widetilde{A}_{L S_{\delta}}\left(\widetilde{\alpha}_{1}\right)=\widetilde{A}_{L S_{\delta}}\left(\widetilde{\alpha}_{2}\right)=0.25+0.45 \cdot \delta$, respectively. 
The example above manifests that the application of the reviewed score degree and accuracy degree functions are unable to make a clear decision between two alternatives with final evaluation represented with apparently different IVIFNs, as they treat them as equal in terms of ordering. This drawback could be overcome by implementing the score degree and accuracy degree functions takeing into account of the decision maker's risk attitude, as proposed in the following section.

\section{The risk attitudinal expected score and accuracy functions}

Yager [41] pointed out that the comparison of FNs is a problem that has been extensively studied and that there is no unique best approach. Indeed, the set of fuzzy numbers is not totally ordered, and therefore a widely used approach to rank FNs consists in converting them into a representative crisp value, and perform the comparison on them [41, 43], a methodology originally proposed by Zadeh in [44]. Recently, a study by Brunelli and Mezei [45] that compares different ranking methods for fuzzy numbers concludes that 'it is impossible to give a final answer to the question on what ranking method is the best. Most of the time choosing a method rather than another is a matter of preference or is context dependent.' Recall that FNs are particular cases of IVIFNs. Thus, the same conclusion applies to IVIFNs. In any case, it seems appropriate to take into account the decision maker's risk attitude to derive a final solution to the MADM problem. In the following, we propose the risk attitudinal expected score and accuracy functions for IVIFNs, which extend the functions reviewed in Section 2.

\subsection{The attitudinal expected function and its properties}

In the following, an interval valued score function for IVIFNs is introduced and a novel expected score function that takes into account the decision maker's attitude via the application of the concept of attitudinal character of a BUM and the continuous ordered weighted average (COWA) operator introduced by Yager [41]. A set of properties of the attitudinal expected score function is also proved. To do this, we start by recalling the concept of a basic unit-monotonic (BUM) function [46]:

Definition 6 (BUM function). A function $Q:[0,1] \rightarrow[0,1]$ such that $Q(0)=0$, $Q(1)=1$ and $Q(x) \geq Q(y)$ if $x \geq y$ is called a basic unit-monotonic (BUM) function 
Given a BUM function, $Q$, Yager proposed the following definition of the COWA operators [41]:

Definition 7 (COWA Operator). Let $I N T(\mathbb{R})$ be the set of all closed subintervals of $\mathbb{R}$. A continuous ordered weighted average (COWA) operator is a mapping $F_{Q}: \operatorname{INT}(\mathbb{R}) \rightarrow$ $\mathbb{R}$ which has an associated BUM function, $Q$, such that

$$
F_{Q}([a, b])=(1-\lambda) \cdot a+\lambda \cdot b
$$

where

$$
\lambda=\int_{0}^{1} Q(y) d y .
$$

is the attitudinal character of $Q$. Thus, $F_{Q}([a, b])$ is the weighted average of the end points of the closed interval with attitudinal character parameter $\lambda$, and it is known as the attitudinal expected value of $[a, b]$.

In the following, an interval-valued score function is proposed for IVIFNs to derive, after the application of expression (12), the new attitudinal expected score function to be applied in the resolution of interval-valued intuitionistic fuzzy MADM problems:

Definition 8 (IVIFN Interval-Valued Score Function). Let $\operatorname{INT}([-1,1])$ be the set of all closed subintervals of $[-1,1]$. The interval-valued score function associated to an IVIFN $\widetilde{\alpha}(x)=\left(\left[\mu^{-}(x), \mu^{+}(x)\right],\left[\nu^{-}(x), \nu^{+}(x)\right]\right)$ is given as follows:

$$
\begin{gathered}
\widetilde{S}_{W C}(\widetilde{\alpha}): X \longrightarrow \operatorname{INT}([-1,1]), \\
\widetilde{S}_{W C}(\widetilde{\alpha})(x)=\left[\mu^{-}(x), \mu^{+}(x)\right]-\left[\nu^{-}(x), \nu^{+}(x)\right]=\left[\mu^{-}(x)-\nu^{+}(x), \mu^{+}(x)-\nu^{-}(x)\right]
\end{gathered}
$$

As it was done before, we will drop the symbol $x$ when referring to the interval-valued score function of an IVIFN $\widetilde{\alpha}$, and it will be denoted simply as $\widetilde{S}_{W C}(\widetilde{\alpha})=\left[\mu^{-}-\nu^{+}, \mu^{+}-\right.$ $\left.\nu^{-}\right]$.

Definition 9 (IVIFN Attitudinal Expected Score Function). The attitudinal expected score function associated to an IVIFN $\widetilde{\alpha}, \widetilde{S}_{W C}$ is

$$
E\left(\widetilde{S}_{W C}(\widetilde{\alpha})\right)_{\lambda}=(1-\lambda) \cdot\left(\mu^{-}-\nu^{+}\right)+\lambda \cdot\left(\mu^{+}-\nu^{-}\right)
$$

where $\lambda$ is the attitudinal character of a BUM function $Q$. 
Notice that because the attitudinal expected score function is based on the COWA operator, some of the properties of this last one are expected to be inherited by the former one. In the following, we provide such set of properties.

The attitudinal expected score function is monotonic with respect to the containment relation of IVIFNs:

Proposition 6. Given any two IVIFNs $\widetilde{\alpha}_{1}=\left(\left[\mu_{1}^{-}, \mu_{1}^{+}\right],\left[\nu_{1}^{-}, \nu_{1}^{+}\right]\right)$and $\widetilde{\alpha}_{2}=\left(\left[\mu_{2}^{-}, \mu_{2}^{+}\right],\left[\nu_{2}^{-}, \nu_{2}^{+}\right]\right)$, such that

$$
\widetilde{\alpha}_{1} \subseteq \widetilde{\alpha}_{2}
$$

then

$$
E\left(\widetilde{S}_{W C}\left(\widetilde{\alpha}_{1}\right)\right)_{\lambda} \leq E\left(\widetilde{S}_{W C}\left(\widetilde{\alpha}_{2}\right)\right)_{\lambda} .
$$

Proof. According to Definition 9, we have

$E\left(\widetilde{S}_{W C}\left(\widetilde{\alpha}_{1}\right)\right)_{\lambda}-E\left(\widetilde{S}_{W C}\left(\widetilde{\alpha}_{2}\right)\right)_{\lambda}=(1-\lambda) \cdot\left[\left(\mu_{1}^{-}-\nu_{1}^{+}\right)-\left(\mu_{2}^{-}-\nu_{2}^{+}\right)\right]+\lambda \cdot\left[\left(\mu_{1}^{+}-\nu_{1}^{-}\right)-\left(\mu_{2}^{+}-\nu_{2}^{-}\right)\right]$

Because $\widetilde{\alpha}_{1} \subseteq \widetilde{\alpha}_{2}$ then it is $\mu_{1}^{-} \leq \mu_{2}^{-}, \mu_{1}^{+} \leq \mu_{2}^{+}, \nu_{1}^{-} \geq \nu_{2}^{-}$, and $\nu_{1}^{+} \geq \nu_{2}^{+}$, and therefore

$$
\mu_{1}^{-}-\nu_{1}^{+} \leq \mu_{2}^{-}-\nu_{2}^{+} \wedge \mu_{1}^{+}-\nu_{1}^{-} \leq \mu_{2}^{+}-\nu_{2}^{-} \text {. }
$$

Thus, we conclude:

$$
E\left(\widetilde{S}_{W C}\left(\widetilde{\alpha}_{1}\right)\right)_{\lambda}-E\left(\widetilde{S}_{W C}\left(\widetilde{\alpha}_{2}\right)\right)_{\lambda} \leq 0
$$

Notice that fixing an IVIFN $\widetilde{\alpha}$ the attitudinal expected score function can be consider a function of the attitudinal character $\lambda$, of which it exhibits the following type of monotonicity property.

Proposition 7. Given an IVIFN, $\widetilde{\alpha}=\left(\left[\mu^{-}, \mu^{+}\right],\left[\nu^{-}, \nu^{+}\right]\right)$, the attitudinal expected score function $E\left(\widetilde{S}_{W C}(\widetilde{\alpha})\right)_{\lambda}$ is monotonic with respect to the argument $\lambda$, i.e.

$$
\lambda_{1} \geq \lambda_{2} \Rightarrow E\left(\widetilde{S}_{W C}(\widetilde{\alpha})\right)_{\lambda_{1}} \geq E\left(\widetilde{S}_{W C}(\widetilde{\alpha})\right)_{\lambda_{2}}
$$

Proof. Notice that $E\left(\widetilde{S}_{W C}(\widetilde{\alpha})\right)_{\lambda}$ can be re-written as follows

$$
E\left(\widetilde{S}_{W C}(\widetilde{\alpha})\right)_{\lambda}=\left(\mu^{-}-\nu^{+}\right)+\lambda \cdot\left(\mu^{+}-\mu^{-}+\nu^{+}-\nu^{-}\right)
$$

Because $\mu^{+}-\mu^{-}+\nu^{+}-\nu^{-} \geq 0$ it is obvious that $E\left(\widetilde{S}_{W C}(\widetilde{\alpha})\right)_{\lambda}$ is increasing with respect to $\lambda$. 
The attitudinal expected score function is also bounded.

Proposition 8. Given an IVIFN $\widetilde{\alpha}=\left(\left[\mu^{-}, \mu^{+}\right],\left[\nu^{-}, \nu^{+}\right]\right)$, we have

$$
-1 \leq \mu^{-}-\nu^{+} \leq E\left(\widetilde{S}_{W C}(\widetilde{\alpha})\right)_{\lambda} \leq \mu^{+}-\nu^{-} \leq 1
$$

Proof. From Proposition 7 we know that the minimum and maximum values of $E\left(\widetilde{S}_{W C}(\widetilde{\alpha})\right)_{\lambda}$ are obtained for $\lambda=0$ and $\lambda=1$, respectively. We have:

$$
\begin{aligned}
& E\left(\widetilde{S}_{W C}(\widetilde{\alpha})\right)_{0}=\mu^{-}-\nu^{+} \\
& E\left(\widetilde{S}_{W C}(\widetilde{\alpha})\right)_{1}=\mu^{+}-\nu^{-}
\end{aligned}
$$

This completes the proof.

From the results of the preceding theorems, we see that the attitudinal expected score function is a mean operator. The following result proves that the attitudinal expected score function is additive and therefore it is a weighted averaging operator.

Proposition 9. Given any two IVIFNs $\widetilde{\alpha}_{1}=\left(\left[\mu_{1}^{-}, \mu_{1}^{+}\right],\left[\nu_{1}^{-}, \nu_{1}^{+}\right]\right)$and $\widetilde{\alpha}_{2}=\left(\left[\mu_{2}^{-}, \mu_{2}^{+}\right],\left[\nu_{2}^{-}, \nu_{2}^{+}\right]\right)$, we have

$$
E\left(\widetilde{S}_{W C}\left(\widetilde{\alpha}_{1}\right)+\widetilde{S}_{W C}\left(\widetilde{\alpha}_{2}\right)\right)_{\lambda}=E\left(\widetilde{S}_{W C}\left(\widetilde{\alpha}_{1}\right)\right)_{\lambda}+E\left(\widetilde{S}_{W C}\left(\widetilde{\alpha}_{2}\right)\right)_{\lambda}
$$

Proof. Because $\widetilde{S}_{W C}\left(\widetilde{\alpha}_{1}\right)+\widetilde{S}_{W C}\left(\widetilde{\alpha}_{2}\right)=\left[\left(\mu_{1}^{-}-\nu_{1}^{+}\right)+\left(\mu_{2}^{-}-\nu_{2}^{+}\right),\left(\mu_{1}^{+}-\nu_{1}^{-}\right)+\left(\mu_{2}^{+}-\nu_{2}^{-}\right)\right]$ then it is

$$
E\left(\widetilde{S}_{W C}\left(\widetilde{\alpha}_{1}\right)+\widetilde{S}_{W C}\left(\widetilde{\alpha}_{2}\right)\right)_{\lambda}=(1-\lambda) \cdot\left[\left(\mu_{1}^{-}-\nu_{1}^{+}\right)+\left(\mu_{2}^{-}-\nu_{2}^{+}\right)\right]+\lambda \cdot\left[\left(\mu_{1}^{+}-\nu_{1}^{-}\right)+\left(\mu_{2}^{+}-\nu_{2}^{-}\right)\right]
$$

Re-arranging the right-hand side we have

$$
\begin{aligned}
E\left(\widetilde{S}_{W C}\left(\widetilde{\alpha}_{1}\right)+\widetilde{S}_{W C}\left(\widetilde{\alpha}_{2}\right)\right)_{\lambda}= & {\left[(1-\lambda) \cdot\left(\mu_{1}^{-}-\nu_{1}^{+}\right)+\lambda \cdot\left(\mu_{1}^{+}-\nu_{1}^{-}\right)\right] } \\
& +\left[(1-\lambda) \cdot\left(\mu_{2}^{-}-\nu_{2}^{+}\right)+\lambda \cdot\left(\mu_{2}^{+}-\nu_{2}^{-}\right)\right.
\end{aligned}
$$

Thus:

$$
E\left(\widetilde{S}_{W C}\left(\widetilde{\alpha}_{1}\right)+\widetilde{S}_{W C}\left(\widetilde{\alpha}_{2}\right)\right)_{\lambda}=E\left(\widetilde{S}_{W C}\left(\widetilde{\alpha}_{1}\right)\right)_{\lambda}+E\left(\widetilde{S}_{W C}\left(\widetilde{\alpha}_{2}\right)\right)_{\lambda}
$$

The attitudinal expected score function generalises $\mathrm{Xu}$ and Chen's score function as the following result proves: 
Proposition 10. The IVIFN score function $\widetilde{S}_{X C}$ and the IVIFN attitudinal expected score function $E\left(\widetilde{S}_{W C}(\widetilde{\alpha})\right)_{\lambda}$ are related as follows

$$
E\left(\widetilde{S}_{W C}(\widetilde{\alpha})\right)_{0.5}=\widetilde{S}_{X C}(\widetilde{\alpha})
$$

Proof. When $\lambda=0.5$ the expression of $E\left(\widetilde{S}_{W C}(\widetilde{\alpha})\right)_{\lambda}$ reduces to

$$
E\left(\widetilde{S}_{W C}(\widetilde{\alpha})\right)_{0.5}=\frac{1}{2} \cdot\left(\mu^{-}-\nu^{+}\right)+\frac{1}{2} \cdot\left(\mu^{+}-\nu^{-}\right)=\frac{\mu^{-}+\mu^{-}-\nu^{-}-\nu^{-}}{2}=\widetilde{S}_{X C}(\widetilde{\alpha}) .
$$

In the following we will provide a sensitivity analysis of the attitudinal expected score function with respect to the attitudinal character $\lambda$, i.e we will provide the conditions under which the ordering of two IVIFNs is not affected by a change in the attitudinal parameter.

Theorem 1. Let $\widetilde{\alpha}_{i}=\left(\left[\mu_{i}^{-}, \mu_{i}^{+}\right],\left[\nu_{i}^{-}, \nu_{i}^{+}\right]\right)$and $\widetilde{\alpha}_{j}=\left(\left[\mu_{j}^{-}, \mu_{j}^{+}\right],\left[\nu_{j}^{-}, \nu_{j}^{+}\right]\right)$be two IVIFNs, and let $\lambda$ be the attitudinal parameter associated to BUM function $Q$ under which it has been established that

$$
E\left(\widetilde{S}_{W C}\left(\widetilde{\alpha}_{i}\right)\right)_{\lambda} \leq E\left(\widetilde{S}_{W C}\left(\widetilde{\alpha}_{j}\right)\right)_{\lambda}
$$

Let $\Delta \lambda$ be a perturbation of the attitudinal character $\lambda$ with $0 \leq \lambda+\Delta \lambda \leq 1$. Then we have

$$
E\left(\widetilde{S}_{W C}\left(\widetilde{\alpha}_{i}\right)\right)_{\lambda+\Delta \lambda} \leq E\left(\widetilde{S}_{W C}\left(\widetilde{\alpha}_{j}\right)\right)_{\lambda+\Delta \lambda}
$$

iff

$$
\begin{cases}\max \left\{-\lambda, \frac{E\left(\widetilde{S}_{W C}\left(\widetilde{\alpha}_{j}\right)\right)_{\lambda}-E\left(\widetilde{S}_{W C}\left(\widetilde{\alpha}_{i}\right)\right)_{\lambda}}{\beta_{i}-\beta_{j}}\right\} \leq \Delta \lambda \leq 1-\lambda, & \text { if } \beta_{i}<\beta_{j} \\ -\lambda \leq \Delta \lambda \leq 1-\lambda, & \text { if } \beta_{j}=\beta_{i} \\ -\lambda \leq \Delta \lambda \leq \min \left\{1-\lambda, \frac{E\left(\widetilde{S}_{W C}\left(\widetilde{\alpha}_{j}\right)\right)_{\lambda}-E\left(\widetilde{S}_{W C}\left(\widetilde{\alpha}_{i}\right)\right)_{\lambda}}{\beta_{i}-\beta_{j}}\right\}, & \text { if } \beta_{i}>\beta_{j}\end{cases}
$$

where $\beta_{i}=\mu_{i}^{+}-\mu_{i}^{-}+\nu_{i}^{+}-\nu_{i}^{-}$and $\beta_{j}=\mu_{j}^{+}-\mu_{j}^{-}+\nu_{j}^{+}-\nu_{j}^{-}$.

Proof. Firstly, we note that $\Delta \lambda$ is subject to the following constraint:

$$
-\lambda \leq \Delta \lambda \leq 1-\lambda
$$


We have the following relation between $E\left(\widetilde{S}_{W C}\left(\widetilde{\alpha}_{i}\right)\right)_{\lambda+\Delta \lambda}$ and $E\left(\widetilde{S}_{W C}\left(\widetilde{\alpha}_{i}\right)\right)_{\lambda}$ :

$$
E\left(\widetilde{S}_{W C}\left(\widetilde{\alpha}_{i}\right)\right)_{\lambda+\Delta \lambda}=E\left(\widetilde{S}_{W C}\left(\widetilde{\alpha}_{i}\right)\right)_{\lambda}+\Delta \lambda \cdot \beta_{i}
$$

where $\beta_{i}=\mu_{i}^{+}-\mu_{i}^{-}+\nu_{i}^{+}-\nu_{i}^{-}$. The following equivalence holds:

$$
E\left(\widetilde{S}_{W C}\left(\widetilde{\alpha}_{i}\right)\right)_{\lambda+\Delta \lambda} \leq E\left(\widetilde{S}_{W C}\left(\widetilde{\alpha}_{j}\right)\right)_{\lambda+\Delta \lambda} \Leftrightarrow \Delta \lambda \cdot\left(\beta_{i}-\beta_{j}\right) \leq E\left(\widetilde{S}_{W C}\left(\widetilde{\alpha}_{j}\right)\right)_{\lambda}-E\left(\widetilde{S}_{W C}\left(\widetilde{\alpha}_{i}\right)\right)_{\lambda}
$$

Three scenarios are possible:

- $\beta_{i}=\beta_{j}$. Because $E\left(\widetilde{S}_{W C}\left(\widetilde{\alpha}_{i}\right)\right)_{\lambda} \leq E\left(\widetilde{S}_{W C}\left(\widetilde{\alpha}_{j}\right)\right)_{\lambda}$ then $(17)$ is true for any value of $\Delta \lambda$, i.e.

$$
-\lambda \leq \Delta \lambda \leq 1-\lambda
$$

- $\beta_{i}>\beta_{j} \Leftrightarrow \Delta \lambda \leq \frac{E\left(\widetilde{S}_{W C}\left(\widetilde{\alpha}_{j}\right)\right)_{\lambda}-E\left(\widetilde{S}_{W C}\left(\widetilde{\alpha}_{i}\right)\right)_{\lambda}}{\beta_{i}-\beta_{j}}$, and therefore:

$$
-\lambda \leq \Delta \lambda \leq \min \left\{1-\lambda, \frac{E\left(\widetilde{S}_{W C}\left(\widetilde{\alpha}_{j}\right)\right)_{\lambda}-E\left(\widetilde{S}_{W C}\left(\widetilde{\alpha}_{i}\right)\right)_{\lambda}}{\beta_{i}-\beta_{j}}\right\} .
$$

- $\beta_{i}<\beta_{j} \Leftrightarrow \Delta \lambda \geq \frac{E\left(\widetilde{S}_{W C}\left(\widetilde{\alpha}_{j}\right)\right)_{\lambda}-E\left(\widetilde{S}_{W C}\left(\widetilde{\alpha}_{i}\right)\right)_{\lambda}}{\beta_{i}-\beta_{j}}$, and therefore:

$$
\max \left\{-\lambda, \frac{E\left(\widetilde{S}_{W C}\left(\widetilde{\alpha}_{j}\right)\right)_{\lambda}-E\left(\widetilde{S}_{W C}\left(\widetilde{\alpha}_{i}\right)\right)_{\lambda}}{\beta_{i}-\beta_{j}}\right\} \leq \Delta \lambda \leq 1-\lambda .
$$

\subsection{The attitudinal expected accuracy function and its properties}

Following the methodology of Section 3.1, an interval valued accuracy function for IVIFNs and a novel expected accuracy function that takes into account the decision maker's attitude are introduced.

Definition 10 (IVIFN Interval-Valued Accuarcy Function). Let $I N T([0,1])$ be the set of all closed subintervals of $[0,1]$. The interval-valued accuracy function associated to an IVIFN, $\widetilde{\alpha}(x)=\left(\left[\mu^{-}(x), \mu^{+}(x)\right],\left[\nu^{-}(x), \nu^{+}(x)\right]\right)$ is given as follows

$$
\widetilde{A}_{W C}(\widetilde{\alpha}): X \longrightarrow \operatorname{INT}([0,1])
$$




$$
\widetilde{A}_{W C}(\widetilde{\alpha})(x)=\left[\mu^{-}(x), \mu^{+}(x)\right]+\left[\nu^{-}(x), \nu^{+}(x)\right]=\left[\mu^{-}(x)+\nu^{-}(x), \mu^{+}(x)+\nu^{+}(x)\right]
$$

Again, we will drop the symbol $x$ when referring to the interval-valued accuracy function of an IVIFN $\widetilde{\alpha}$, and it will be denoted simply as $\widetilde{A}_{W C}(\widetilde{\alpha})=\left[\mu^{-}+\nu^{-}, \mu^{+}+\nu^{+}\right]$.

Definition 11 (IVIFN Attitudinal Expected Accuarcy Function). The attitudinal expected accuracy function associated to an IVIFN $\widetilde{\alpha}, \widetilde{A}_{W C}$ is

$$
E\left(\widetilde{A}_{W C}(\widetilde{\alpha})\right)_{\lambda}=(1-\lambda) \cdot\left(\mu^{-}+\nu^{-}\right)+\lambda \cdot\left(\mu^{+}+\nu^{+}\right)
$$

where $\lambda$ is the attitudinal character of a BUM function $Q$.

As with the attitudinal expected score function, the attitudinal expected accuracy function verifies some kind of monotonicity property with respect to the IVIFN argument.

Proposition 11. Given two IVIFNs, $\widetilde{\alpha}_{1}=\left(\left[\mu_{1}^{-}, \mu_{1}^{+}\right],\left[\nu_{1}^{-}, \nu_{1}^{+}\right]\right)$and $\widetilde{\alpha}_{2}=\left(\left[\mu_{2}^{-}, \mu_{2}^{+}\right],\left[\nu_{2}^{-}, \nu_{2}^{+}\right]\right)$, such that

$$
\mu_{1}^{-}+\nu_{1}^{-} \leq \mu_{2}^{-}+\nu_{2}^{-} \wedge \mu_{1}^{+}+\nu_{1}^{+} \leq \mu_{2}^{+}+\nu_{2}^{+}
$$

then

$$
E\left(\widetilde{A}_{W C}\left(\widetilde{\alpha}_{1}\right)\right)_{\lambda} \leq E\left(\widetilde{A}_{W C}\left(\widetilde{\alpha}_{2}\right)\right)_{\lambda} .
$$

Proof. Obvious

In Proposition 11, it is sufficient that $\mu_{1}^{-} \leq \mu_{2}^{-}, \nu_{1}^{-} \leq \nu_{2}^{-}, \mu_{1}^{+} \leq \mu_{2}^{+}$and $\nu_{1}^{+} \leq \nu_{2}^{+}$to ensure that $E\left(\widetilde{A}_{W C}\left(\widetilde{\alpha}_{1}\right)\right)_{\lambda} \leq E\left(\widetilde{A}_{W C}\left(\widetilde{\alpha}_{2}\right)\right)_{\lambda}$ is true.

Notice that $E\left(\widetilde{A}_{W C}(\widetilde{\alpha})\right)_{\lambda}$ can be re-written as follows:

$$
E\left(\widetilde{A}_{W C}(\widetilde{\alpha})\right)_{\lambda}=\left(\mu^{-}+\nu^{-}\right)+\lambda \cdot\left(\mu^{+}-\mu^{-}+\nu^{+}-\nu^{-}\right)=E\left(\widetilde{S}_{W C}(\widetilde{\alpha})\right)_{\lambda}+\left(\nu^{+}+\nu^{-}\right) .
$$

Therefore, the following relationship between the attitudinal expected accuracy score function and the attitudinal expected accuracy function is proved:

Proposition 12. Given an IVIFN $\widetilde{\alpha}=\left(\left[\mu^{-}, \mu^{+}\right],\left[\nu^{-}, \nu^{+}\right]\right)$, the following holds:

$$
E\left(\widetilde{S}_{W C}(\widetilde{\alpha})\right)_{\lambda} \leq E\left(\widetilde{A}_{W C}(\widetilde{\alpha})\right)_{\lambda}
$$

An immediate consequence of expression (20) is that the attitudinal expected score function properties of monotonicity with respect to the argument $\lambda$, boundedness and additivity are also verified by the attitudinal expected accuarcy function. 
Proposition 13. Given an IVIFN $\widetilde{\alpha}=\left(\left[\mu^{-}, \mu^{+}\right],\left[\nu^{-}, \nu^{+}\right]\right)$, the attitudinal expected accuarcy function $E\left(\widetilde{A}_{W C}(\widetilde{\alpha})\right)_{\lambda}$ is monotonic with respect to the argument $\lambda$, i.e.

$$
\lambda_{1} \geq \lambda_{2} \Rightarrow E\left(\widetilde{A}_{W C}(\widetilde{\alpha})\right)_{\lambda_{1}} \geq E\left(\widetilde{A}_{W C}(\widetilde{\alpha})\right)_{\lambda_{2}}
$$

Proposition 14. Given an IVIFN $\widetilde{\alpha}=\left(\left[\mu^{-}, \mu^{+}\right],\left[\nu^{-}, \nu^{+}\right]\right)$, we have

$$
0 \leq \mu^{-}+\nu^{-} \leq E\left(\widetilde{A}_{W C}(\widetilde{\alpha})\right)_{\lambda} \leq \mu^{+}+\nu^{+} \leq 1
$$

Proposition 15. Given any two IVIFNs $\widetilde{\alpha}_{1}=\left(\left[\mu_{1}^{-}, \mu_{1}^{+}\right],\left[\nu_{1}^{-}, \nu_{1}^{+}\right]\right)$and $\widetilde{\alpha}_{2}=\left(\left[\mu_{2}^{-}, \mu_{2}^{+}\right],\left[\nu_{2}^{-}, \nu_{2}^{+}\right]\right)$, we have

$$
E\left(\widetilde{A}_{W C}\left(\widetilde{\alpha}_{1}\right)+\widetilde{A}_{W C}\left(\widetilde{\alpha}_{2}\right)\right)_{\lambda}=E\left(\widetilde{A}_{W C}\left(\widetilde{\alpha}_{1}\right)\right)_{\lambda}+E\left(\widetilde{A}_{W C}\left(\widetilde{\alpha}_{2}\right)\right)_{\lambda}
$$

Additionally, the attitudinal expected accuracy function verifies a type of symmetry property with respect to the interval-valued membership and non-membership degrees of the IVIFN argument.

Proposition 16. Given an IVIFN $\widetilde{\alpha}=\left(\left[\mu^{-}, \mu^{+}\right],\left[\nu^{-}, \nu^{+}\right]\right)$, we have

$$
E\left(\widetilde{A}_{W C}(\widetilde{\alpha})\right)_{\lambda}=E\left(\widetilde{A}_{W C}(\overline{\widetilde{\alpha}})\right)_{\lambda}
$$

Proof. Recall that $\overline{\widetilde{\alpha}}=\left(\left[\nu^{-}, \nu^{+}\right],\left[\mu^{-}, \mu^{+}\right]\right)$, therefore we have

$$
E\left(\widetilde{A}_{W C}(\overline{\widetilde{\alpha}})\right)_{\lambda}=(1-\lambda) \cdot\left(\nu^{-}+\mu^{-}\right)+\lambda \cdot\left(\nu^{+}+\mu^{+}\right)
$$

This expression coincides with (19).

As it happened before with the attitudinal expected score function and Xu and Chen's score function, we have the following result that proves that the attitudinal expected accuracy function extends Xu and Chen's accuracy function:

Proposition 17. The IVIFN accuracy function $\widetilde{A}_{X C}$ and the IVIFN attitudinal expected accuracy function $E\left(\widetilde{A}_{W C}(\widetilde{\alpha})\right)_{\lambda}$ are related as follows

$$
E\left(\widetilde{A}_{W C}(\widetilde{\alpha})\right)_{0.5}=\widetilde{A}_{X C}(\widetilde{\alpha})
$$

We observe that Proposition 5 applies to $E\left(\widetilde{S}_{W C}(\widetilde{\alpha})\right)_{\lambda}$ and $E\left(\widetilde{A}_{W C}(\widetilde{\alpha})\right)_{\lambda}$ when $\lambda=0.5$. 
The attitudinal expected accuracy functions $E\left(\widetilde{A}_{W C}\left(\widetilde{\alpha}_{i}\right)\right)_{\lambda+\Delta \lambda}$ and $E\left(\widetilde{A}_{W C}\left(\widetilde{\alpha}_{i}\right)\right)_{\lambda}$ are related as follows:

$$
E\left(\widetilde{A}_{W C}\left(\widetilde{\alpha}_{i}\right)\right)_{\lambda+\Delta \lambda}=E\left(\widetilde{A}_{W C}\left(\widetilde{\alpha}_{i}\right)\right)_{\lambda}+\Delta \lambda \cdot \beta_{i}
$$

where $\beta_{i}=\mu_{i}^{+}-\mu_{i}^{-}+\nu_{i}^{+}-\nu_{i}^{-}$. Therefore, the proof of Theorem 1 is applicable to prove the following result regarding the sensitivity analysis of the attitudinal expected accuracy function with respect to the attitudinal parameter $\lambda$ :

Theorem 2. Let $\widetilde{\alpha}_{i}=\left(\left[\mu_{i}^{-}, \mu_{i}^{+}\right],\left[\nu_{i}^{-}, \nu_{i}^{+}\right]\right)$and $\widetilde{\alpha}_{j}=\left(\left[\mu_{j}^{-}, \mu_{j}^{+}\right],\left[\nu_{j}^{-}, \nu_{j}^{+}\right]\right)$be two IVIFNs, and let $\lambda$ be the attitudinal parameter associated to BUM function $Q$ under which it has been established that

$$
E\left(\widetilde{A}_{W C}\left(\widetilde{\alpha}_{i}\right)\right)_{\lambda} \leq E\left(\widetilde{A}_{W C}\left(\widetilde{\alpha}_{j}\right)\right)_{\lambda} .
$$

Let $\Delta \lambda$ be a perturbation of the attitudinal character $\lambda$ with $0 \leq \lambda+\Delta \lambda \leq 1$. Then we have

$$
E\left(\widetilde{A}_{W C}\left(\widetilde{\alpha}_{i}\right)\right)_{\lambda+\Delta \lambda} \leq E\left(\widetilde{A}_{W C}\left(\widetilde{\alpha}_{j}\right)\right)_{\lambda+\Delta \lambda}
$$

iff

$$
\begin{cases}\max \left\{-\lambda, \frac{\left.E\left(\widetilde{A}_{W C}\left(\widetilde{\alpha}_{j}\right)\right)_{\lambda}-E\left(\widetilde{A}_{W C}\left(\widetilde{\alpha}_{i}\right)\right)_{\lambda}\right\} \leq \Delta \lambda \leq 1-\lambda,}{\beta_{i}-\beta_{j}} \text { if } \beta_{i}<\beta_{j}\right. \\ -\lambda \leq \Delta \lambda \leq 1-\lambda, & \text { if } \beta_{j}=\beta_{i} \\ -\lambda \leq \Delta \lambda \leq \min \left\{1-\lambda, \frac{\left.E\left(\widetilde{A}_{W C}\left(\widetilde{\alpha}_{j}\right)\right)_{\lambda}-E\left(\widetilde{A}_{W C}\left(\widetilde{\alpha}_{i}\right)\right)_{\lambda}\right\},}{\beta_{i}-\beta_{j}},\right. & \text { if } \beta_{i}>\beta_{j}\end{cases}
$$

where $\beta_{i}=\mu_{i}^{+}-\mu_{i}^{-}+\nu_{i}^{+}-\nu_{i}^{-}$and $\beta_{j}=\mu_{j}^{+}-\mu_{j}^{-}+\nu_{j}^{+}-\nu_{j}^{-}$.

\subsection{Ordering relation of interval-valued intuitionistic fuzzy numbers}

Example 1 demonstrates that a two level ordering relation based on the score function and accuracy function developed up to know is insufficient to discriminate correctly between different IVIFNs. The reason for this is that an IVIFN is completely characterised by four parameters in the unit interval subject to a constraint on two of the parameters (the upper membership and non-membership degrees) that reduces the degrees of freedom from four to three. Therefore, it seems as if a three level ordering relation might be needed to accurately identified equality of IVIFNs, and therefore to properly discriminate between different IVIFNs. To achieve this, a new index function is used in conjunction 
with the attitudinal expected score function and the attitudinal expected accuracy function previously developed, which was introduced by Wang et al. in [31] and that is presented in the following definition:

Definition 12 (Membership Uncertainty Index Function). The membership uncertainty index function of an IVIFN, $\widetilde{\alpha}(x)=\left(\left[\mu^{-}(x), \mu^{+}(x)\right],\left[\nu^{-}(x), \nu^{+}(x)\right]\right)$ is given as follows

$$
\begin{gathered}
T(\widetilde{\alpha}): X \longrightarrow[-1,1], \\
T(\widetilde{\alpha})(x)=\left(\mu^{+}(x)-\mu^{-}(x)\right)-\left(\nu^{+}(x)-\nu^{-}(x)\right)
\end{gathered}
$$

For simplicity and consistency with the previous defined functions, we will drop the symbol $x$ when referring to the membership uncertainty index function of an IVIFN $\widetilde{\alpha}$, and it will be denoted simply as $T(\widetilde{\alpha})=\left(\mu^{+}-\mu^{-}\right)-\left(\nu^{+}-\nu^{-}\right)$.

Atanassov and Gargov's defintion of equality of IVIFNs is the following [4]:

Definition 13 (Equality of IVIFNs). Given two IVIFNs, $\widetilde{\alpha}_{1}=\left(\left[\mu_{1}^{-}, \mu_{1}^{+}\right],\left[\nu_{1}^{-}, \nu_{1}^{+}\right]\right)$ and $\widetilde{\alpha}_{2}=\left(\left[\mu_{2}^{-}, \mu_{2}^{+}\right],\left[\nu_{2}^{-}, \nu_{2}^{+}\right]\right)$, we have the following equivalence:

$$
\widetilde{\alpha}_{1}=\widetilde{\alpha}_{2} \Longleftrightarrow \mu_{1}^{-}=\mu_{2}^{-} \wedge \mu_{1}^{+}=\mu_{2}^{+} \wedge \nu_{1}^{-}=\nu_{2}^{-} \wedge \nu_{1}^{+}=\nu_{2}^{+}
$$

Next, we characterise the equality of IVIFNs using the above three functions:

Proposition 18 (Characterisation of Equality of IVIFNs). Given two IVIFNs, $\widetilde{\alpha}_{1}=$ $\left(\left[\mu_{1}^{-}, \mu_{1}^{+}\right],\left[\nu_{1}^{-}, \nu_{1}^{+}\right]\right)$and $\widetilde{\alpha}_{2}=\left(\left[\mu_{2}^{-}, \mu_{2}^{+}\right],\left[\nu_{2}^{-}, \nu_{2}^{+}\right]\right)$, we have the following equivalence:

$$
\begin{gathered}
\widetilde{\alpha}_{1}={\widetilde{\alpha_{2}}} \\
\text { iff } \\
E\left(\widetilde{S}_{W C}\left(\widetilde{\alpha_{1}}\right)\right)_{\lambda}=E\left(\widetilde{S}_{W C}\left(\widetilde{\alpha_{2}}\right)\right)_{\lambda} \wedge E\left(\widetilde{A}_{W C}\left(\widetilde{\alpha_{1}}\right)\right)_{\lambda}=E\left(\widetilde{A}_{W C}\left(\widetilde{\alpha_{2}}\right)\right)_{\lambda} \wedge T\left(\widetilde{\alpha_{1}}\right)=T\left(\widetilde{\alpha_{2}}\right)
\end{gathered}
$$

Proof. We only need to prove that when

$$
E\left(\widetilde{S}_{W C}\left(\widetilde{\alpha_{1}}\right)\right)_{\lambda}=E\left(\widetilde{S}_{W C}\left(\widetilde{\alpha_{2}}\right)\right)_{\lambda} \wedge E\left(\widetilde{A}_{W C}\left(\widetilde{\alpha_{1}}\right)\right)_{\lambda}=E\left(\widetilde{A}_{W C}\left(\widetilde{\alpha_{2}}\right)\right)_{\lambda} \wedge T\left(\widetilde{\alpha_{1}}\right)=T\left(\widetilde{\alpha_{2}}\right)
$$

then it is

$$
\mu_{1}^{-}=\mu_{2}^{-} \wedge \mu_{1}^{+}=\mu_{2}^{+} \wedge \nu_{1}^{-}=\nu_{2}^{-} \wedge \nu_{1}^{+}=\nu_{2}^{+} .
$$


1. $E\left(\widetilde{S}_{W C}\left(\widetilde{\alpha_{1}}\right)\right)_{\lambda}=E\left(\widetilde{S}_{W C}\left(\widetilde{\alpha_{2}}\right)\right)_{\lambda}$ is equivalent to $\left[(1-\lambda) \cdot\left(\mu_{1}^{-}-\nu_{1}^{+}\right)+\lambda \cdot\left(\mu_{1}^{+}-\nu_{1}^{-}\right)\right]=\left[(1-\lambda) \cdot\left(\mu_{2}^{-}-\nu_{2}^{+}\right)+\lambda \cdot\left(\mu_{2}^{+}-\nu_{2}^{-}\right)\right), \forall \lambda \in[0,1]$

Making $\lambda=0$ and $\lambda=1$ we have:

(a) $\mu_{1}^{-}-\nu_{1}^{+}=\mu_{2}^{-}-\nu_{2}^{+}$

(b) $\mu_{1}^{+}-\nu_{1}^{-}=\mu_{2}^{+}-\nu_{2}^{-}$

2. $E\left(\widetilde{A}_{W C}\left(\widetilde{\alpha_{1}}\right)\right)_{\lambda}=E\left(\widetilde{A}_{W C}\left(\widetilde{\alpha_{2}}\right)\right)_{\lambda}$ is equivalent to $\left[(1-\lambda) \cdot\left(\mu_{1}^{-}+\nu_{1}^{-}\right)+\lambda \cdot\left(\mu_{1}^{+}+\nu_{1}^{+}\right)\right]=\left[(1-\lambda) \cdot\left(\mu_{2}^{-}+\nu_{2}^{-}\right)+\lambda \cdot\left(\mu_{2}^{+}+\nu_{2}^{+}\right)\right), \forall \lambda \in[0,1]$.

Making $\lambda=0$ and $\lambda=1$ we have:

(a) $\mu_{1}^{-}+\nu_{1}^{-}=\mu_{2}^{-}+\nu_{2}^{-}$

(b) $\mu_{1}^{+}+\nu_{1}^{+}=\mu_{2}^{+}+\nu_{2}^{+}$

3. $T\left(\widetilde{\alpha_{1}}\right)=T\left(\widetilde{\alpha_{2}}\right)$ is equivalent to

$$
\left(\mu_{1}^{+}-\mu_{1}^{-}\right)-\left(\nu_{1}^{+}-\nu_{1}^{-}\right)=\left(\mu_{2}^{+}-\mu_{2}^{-}\right)-\left(\nu_{2}^{+}-\nu_{2}^{-}\right)
$$

The following expression is computed

$$
2 \cdot 1(\mathrm{a})+2(\mathrm{a})+2(\mathrm{~b})-3
$$

resulting in

$$
4 \cdot \mu_{1}^{-}=4 \cdot \mu_{2}^{-} \Leftrightarrow \mu_{1}^{-}=\mu_{2}^{-} .
$$

Applying this to $1(\mathrm{a})$ and $2(\mathrm{a})$ we have that $\nu_{1}^{+}=\nu_{2}^{+}$and $\nu_{1}^{-}=\nu_{2}^{-}$, respectively. Finally, from $1(\mathrm{~b})$ we derive that $\mu_{1}^{+}=\mu_{2}^{+}$.

This result allows the development of the following order relation on the set of IVIFNs:

Definition 14 (Attitudinal IVIFN Order Relation). Given two IVIFNs, $\widetilde{\alpha}_{1}$ and $\widetilde{\alpha}_{2}$, we say that

$$
\widetilde{\alpha}_{1} \prec \widetilde{\alpha}_{2}
$$

if and only if one the following conditions is true:

1. $E\left(\widetilde{S}_{W C}\left(\widetilde{\alpha_{1}}\right)\right)_{\lambda}<E\left(\widetilde{S}_{W C}\left(\widetilde{\alpha_{2}}\right)\right)_{\lambda}$ 
2. $E\left(\widetilde{S}_{W C}\left(\widetilde{\alpha_{1}}\right)\right)_{\lambda}=E\left(\widetilde{S}_{W C}\left(\widetilde{\alpha_{2}}\right)\right)_{\lambda} \wedge E\left(\widetilde{A}_{W C}\left(\widetilde{\alpha_{1}}\right)\right)_{\lambda}<E\left(\widetilde{A}_{W C}\left(\widetilde{\alpha_{2}}\right)\right)_{\lambda}$

3. $E\left(\widetilde{S}_{W C}\left(\widetilde{\alpha_{1}}\right)\right)_{\lambda}=E\left(\widetilde{S}_{W C}\left(\widetilde{\alpha_{2}}\right)\right)_{\lambda} \wedge E\left(\widetilde{A}_{W C}\left(\widetilde{\alpha_{1}}\right)\right)_{\lambda}=E\left(\widetilde{A}_{W C}\left(\widetilde{\alpha_{2}}\right)\right)_{\lambda} \wedge T\left(\widetilde{\alpha_{1}}\right)>$ $T\left(\widetilde{\alpha_{2}}\right)$

The next result proves that the order relation $\prec$ is a strict order.

Theorem 3 (Strict Order). The relation $\prec$ on the set of IVIFNs is a strict order, i.e. $\prec i s$

1. Irreflexive: $\forall \widetilde{\alpha}: \widetilde{\alpha} \prec \widetilde{\alpha}$ does not hold.

2. Asymmetric: $\forall \widetilde{\alpha}_{1}, \widetilde{\alpha}_{2}:$ if $\widetilde{\alpha}_{1} \prec \widetilde{\alpha}_{2}$, then $\widetilde{\alpha}_{2} \prec \widetilde{\alpha}_{2}$ does not hold.

3. Transitive: $\forall \widetilde{\alpha}_{1}, \widetilde{\alpha}_{2}, \widetilde{\alpha}_{3}:$ if $\widetilde{\alpha}_{1} \prec \widetilde{\alpha}_{2}$ and $\widetilde{\alpha}_{2} \prec \widetilde{\alpha}_{3}$, then $\widetilde{\alpha}_{1} \prec \widetilde{\alpha}_{3}$.

Proof. Items 1. and 2. are obvious from Definition 14 . We prove the transitivity property. Starting with $\widetilde{\alpha}_{1} \prec \widetilde{\alpha}_{2}$, from Definition 14 we have three possible cases:

1. $E\left(\widetilde{S}_{W C}\left(\widetilde{\alpha}_{1}\right)\right)_{\lambda}<E\left(\widetilde{S}_{W C}\left(\widetilde{\alpha}_{2}\right)\right)_{\lambda}$. In this case, it is clear that $E\left(\widetilde{S}_{W C}\left(\widetilde{\alpha}_{1}\right)\right)_{\lambda}<$ $E\left(\widetilde{S}_{W C}\left(\widetilde{\alpha}_{3}\right)\right)_{\lambda}$, no matter which condition is true for $\widetilde{\alpha}_{2} \prec \widetilde{\alpha}_{3}$.

2. $E\left(\widetilde{S}_{W C}\left(\widetilde{\alpha}_{1}\right)\right)_{\lambda}=E\left(\widetilde{S}_{W C}\left(\widetilde{\alpha}_{2}\right)\right)_{\lambda} \wedge E\left(\widetilde{A}_{W C}\left(\widetilde{\alpha}_{1}\right)\right)_{\lambda}<E\left(\widetilde{A}_{W C}\left(\widetilde{\alpha}_{2}\right)\right)_{\lambda}$. Because $\widetilde{\alpha}_{2} \prec \widetilde{\alpha}_{3}$ then one of the following is true:

(a) $E\left(\widetilde{S}_{W C}\left(\widetilde{\alpha}_{2}\right)\right)_{\lambda}<E\left(\widetilde{S}_{W C}\left(\widetilde{\alpha}_{3}\right)\right)_{\lambda}$. In this case, we conclude that $E\left(\widetilde{S}_{W C}\left(\widetilde{\alpha}_{1}\right)\right)_{\lambda}<$ $E\left(\widetilde{S}_{W C}\left(\widetilde{\alpha}_{3}\right)\right)_{\lambda}$ and therefore it is $\widetilde{\alpha}_{1} \prec \widetilde{\alpha}_{3}$.

(b) $E\left(\widetilde{S}_{W C}\left(\widetilde{\alpha}_{2}\right)\right)_{\lambda}=E\left(\widetilde{S}_{W C}\left(\widetilde{\alpha}_{3}\right)\right)_{\lambda} \wedge E\left(\widetilde{A}_{W C}\left(\widetilde{\alpha}_{2}\right)\right)_{\lambda}<E\left(\widetilde{A}_{W C}\left(\widetilde{\alpha}_{3}\right)\right)_{\lambda}$. In this case, we conclude that $E\left(\widetilde{S}_{W C}\left(\widetilde{\alpha}_{1}\right)\right)_{\lambda}=E\left(\widetilde{S}_{W C}\left(\widetilde{\alpha}_{3}\right)\right)_{\lambda} \wedge E\left(\widetilde{A}_{W C}\left(\widetilde{\alpha}_{1}\right)\right)_{\lambda}<$ $E\left(\widetilde{A}_{W C}\left(\widetilde{\alpha}_{3}\right)\right)_{\lambda}$ and therefore it is $\widetilde{\alpha}_{1} \prec \widetilde{\alpha}_{3}$.

(c) $E\left(\widetilde{S}_{W C}\left(\widetilde{\alpha}_{2}\right)\right)_{\lambda}=E\left(\widetilde{S}_{W C}\left(\widetilde{\alpha}_{3}\right)\right)_{\lambda} \wedge E\left(\widetilde{A}_{W C}\left(\widetilde{\alpha}_{2}\right)\right)_{\lambda}=E\left(\widetilde{A}_{W C}\left(\widetilde{\alpha}_{3}\right)\right)_{\lambda} \wedge$ $T\left(\widetilde{\alpha}_{2}\right)>T\left(\widetilde{\alpha}_{3}\right)$. In this case, we conclude that $E\left(\widetilde{S}_{W C}\left(\widetilde{\alpha}_{1}\right)\right)_{\lambda}=E\left(\widetilde{S}_{W C}\left(\widetilde{\alpha}_{3}\right)\right)_{\lambda} \wedge$ $E\left(\widetilde{A}_{W C}\left(\widetilde{\alpha}_{1}\right)\right)_{\lambda}<E\left(\widetilde{A}_{W C}\left(\widetilde{\alpha}_{3}\right)\right)_{\lambda}$ and therefore it is $\widetilde{\alpha}_{1} \prec \widetilde{\alpha}_{3}$.

3. $E\left(\widetilde{S}_{W C}\left(\widetilde{\alpha}_{1}\right)\right)_{\lambda}=E\left(\widetilde{S}_{W C}\left(\widetilde{\alpha}_{2}\right)\right)_{\lambda} \wedge E\left(\widetilde{A}_{W C}\left(\widetilde{\alpha}_{1}\right)\right)_{\lambda}=E\left(\widetilde{A}_{W C}\left(\widetilde{\alpha}_{2}\right)\right)_{\lambda} \wedge T\left(\widetilde{\alpha}_{1}\right)>$ $T\left(\widetilde{\alpha}_{2}\right)$. Because $\widetilde{\alpha}_{2} \prec \widetilde{\alpha}_{3}$ then one of the following is true: 
(a) $E\left(\widetilde{S}_{W C}\left(\widetilde{\alpha}_{2}\right)\right)_{\lambda}<E\left(\widetilde{S}_{W C}\left(\widetilde{\alpha}_{3}\right)\right)_{\lambda}$. In this case, we conclude that $E\left(\widetilde{S}_{W C}\left(\widetilde{\alpha}_{1}\right)\right)_{\lambda}<$ $E\left(\widetilde{S}_{W C}\left(\widetilde{\alpha}_{3}\right)\right)_{\lambda}$ and therefore it is $\widetilde{\alpha}_{1} \prec \widetilde{\alpha}_{3}$.

(b) $E\left(\widetilde{S}_{W C}\left(\widetilde{\alpha}_{2}\right)\right)_{\lambda}=E\left(\widetilde{S}_{W C}\left(\widetilde{\alpha}_{3}\right)\right)_{\lambda} \wedge E\left(\widetilde{A}_{W C}\left(\widetilde{\alpha}_{2}\right)\right)_{\lambda}<E\left(\widetilde{A}_{W C}\left(\widetilde{\alpha}_{3}\right)\right)_{\lambda}$. In this case, we conclude that $E\left(\widetilde{S}_{W C}\left(\widetilde{\alpha}_{1}\right)\right)_{\lambda}=E\left(\widetilde{S}_{W C}\left(\widetilde{\alpha}_{3}\right)\right)_{\lambda} \wedge E\left(\widetilde{A}_{W C}\left(\widetilde{\alpha}_{1}\right)\right)_{\lambda}<$ $E\left(\widetilde{A}_{W C}\left(\widetilde{\alpha}_{3}\right)\right)_{\lambda}$ and therefore it is $\widetilde{\alpha}_{1} \prec \widetilde{\alpha}_{3}$.

(c) $E\left(\widetilde{S}_{W C}\left(\widetilde{\alpha}_{2}\right)\right)_{\lambda}=E\left(\widetilde{S}_{W C}\left(\widetilde{\alpha}_{3}\right)\right)_{\lambda} \wedge E\left(\widetilde{A}_{W C}\left(\widetilde{\alpha}_{2}\right)\right)_{\lambda}=E\left(\widetilde{A}_{W C}\left(\widetilde{\alpha}_{3}\right)\right)_{\lambda} \wedge$ $T\left(\widetilde{\alpha}_{2}\right)>T\left(\widetilde{\alpha}_{3}\right)$. In this case, we conclude that $E\left(\widetilde{S}_{W C}\left(\widetilde{\alpha}_{1}\right)\right)_{\lambda}=E\left(\widetilde{S}_{W C}\left(\widetilde{\alpha}_{3}\right)\right)_{\lambda} \wedge$ $E\left(\widetilde{A}_{W C}\left(\widetilde{\alpha}_{1}\right)\right)_{\lambda}=E\left(\widetilde{A}_{W C}\left(\widetilde{\alpha}_{3}\right)\right)_{\lambda} \wedge T\left(\widetilde{\alpha}_{1}\right)>T\left(\widetilde{\alpha}_{3}\right)$ and therefore it is $\widetilde{\alpha}_{1} \prec \widetilde{\alpha}_{3}$.

We conclude that $\widetilde{\alpha}_{1} \prec \widetilde{\alpha}_{2}$ and $\widetilde{\alpha}_{2} \prec \widetilde{\alpha}_{3}$ implies $\widetilde{\alpha}_{1} \prec \widetilde{\alpha}_{3}$.

Theorem 3 allows the following construction of a total order on the set of IVIFNs:

$$
\widetilde{\alpha}_{1} \preceq \widetilde{\alpha}_{2} \text { if and only if } \widetilde{\alpha}_{1} \prec \widetilde{\alpha}_{2} \vee \widetilde{\alpha}_{1}=\widetilde{\alpha}_{2}
$$

Proposition 6 can be re-written as follows:

Proposition 19. Given any two IVIFNs $\widetilde{\alpha}_{1}=\left(\left[\mu_{1}^{-}, \mu_{1}^{+}\right],\left[\nu_{1}^{-}, \nu_{1}^{+}\right]\right)$and $\widetilde{\alpha}_{2}=\left(\left[\mu_{2}^{-}, \mu_{2}^{+}\right],\left[\nu_{2}^{-}, \nu_{2}^{+}\right]\right)$, the following holds:

$$
\widetilde{\alpha}_{1} \subseteq \widetilde{\alpha}_{2} \Rightarrow \widetilde{\alpha}_{1} \preceq \widetilde{\alpha}_{2}
$$

Notice that the results presented in this section are applicable to constant IVIFNs, i.e. IVIFNs verifying

$$
\left(\left[\widetilde{\mu}_{A L}(x), \widetilde{\mu}_{A U}(x)\right],\left[\widetilde{\nu}_{A L}(x), \widetilde{\nu}_{A U}(x)\right]\right)=\left(\left[\mu^{-}, \mu^{+}\right],\left[\nu^{-}, \nu^{+}\right]\right) \forall x \in X
$$

\section{Interval-valued intuitionistic fuzzy multi-attribute decision-making method based on the attitudinal expected functions}

Let $A=\left\{A_{1}, A_{2}, \ldots, A_{m}\right\}$ be a set of alternatives and a set of criteria $C=\left\{C_{1}, C_{2}, \ldots, C_{n}\right\}$ that have associated an importance weight vector $\mathbf{W}=\left(w_{1}, w_{2}, \ldots, w_{n}\right)$ such that:

$$
w_{j} \geq 0 \wedge \sum_{j=1}^{n} w_{j}=1
$$


Each alternative $A_{i}$ is assessed using the following assessment profile of constant IVIFNs on the set of criteria

$$
\begin{aligned}
& \forall i \in\{1,2, \ldots, m\}: A_{i}=\left\{\left\langle C_{j},\left[\widetilde{\mu}_{i L}\left(C_{j}\right), \widetilde{\mu}_{i U}\left(C_{j}\right)\right],\left[\widetilde{\nu}_{i L}\left(C_{j}\right), \widetilde{\nu}_{i U}\left(C_{j}\right)\right]\right\rangle \mid \forall j \in\{1,2, \ldots, n\}:\right. \\
&\left.C_{j} \in C: 0 \leq \widetilde{\mu}_{i U}\left(C_{j}\right)+\widetilde{\nu}_{i U}\left(C_{j}\right) \leq 1, \widetilde{\mu}_{i L}\left(C_{j}\right) \wedge \widetilde{\nu}_{i L}\left(C_{j}\right) \geq 0\right\}
\end{aligned}
$$

Using the following notation from the previous section

$$
\left(\left[\widetilde{\mu}_{i L}\left(C_{j}\right), \widetilde{\mu}_{i U}\left(C_{j}\right)\right],\left[\widetilde{\nu}_{i L}\left(C_{j}\right), \widetilde{\nu}_{i U}\left(C_{j}\right)\right]\right)=\left(\left[\mu_{i j}^{-}, \mu_{i j}^{+}\right],\left[\nu_{i j}^{-}, \nu_{i j}^{+}\right]\right)=\widetilde{\alpha}_{i j}
$$

An interval-valued intuitionistic fuzzy decision matrix, $\widetilde{D}=\left(\widetilde{\alpha}_{i j}\right)_{m \times n}$, is elicited. The problem here to solve is to produce a final ranking of the alternatives based on the information contained in the interval-valued intuitionistic fuzzy decision matrix.

Example 2. Let us assume a set of four alternatives $\left\{A_{1}, A_{2}, A_{3}, A_{4}\right\}$ that are assessed against a set of four criteria $\left\{C_{1}, C_{2}, C_{3}, C_{4}\right\}$, with corresponding weighting vector $\mathbf{W}=$ $(0.1,0.4,0.4,0.1)$, resulting in the following interval-valued intuitionistic fuzzy decision matrix.

$$
\widetilde{D}=\left(\begin{array}{cccc}
([0.4,0.5],[0.2,0.3]) & ([0.3,0.6],[0.3,0.4]) & ([0.6,0.7],[0.2,0.3]) & ([0.4,0.6],[0.1,0.2]) \\
([0.4,0.7],[0.1,0.2]) & ([0.6,0.7],[0.1,0.2]) & ([0.3,0.6],[0.2,0.3]) & ([0.4,0.5],[0.1,0.4]) \\
([0.3,0.4],[0.2,0.3]) & ([0.4,0.7],[0.2,0.3]) & ([0.4,0.5],[0.1,0.3]) & ([0.6,0.8],[0.1,0.2]) \\
([0.3,0.6],[0.2,0.3]) & ([0.4,0.5],[0.1,0.4]) & ([0.5,0.8],[0.1,0.2]) & ([0.5,0.6],[0.1,0.2])
\end{array}\right)
$$

Each alternative, $A_{i}$, can be associated its collective attitudinal expected score value as the weighted average of its corresponding assessment profile

$$
s_{\lambda}\left(A_{i}\right)=\sum_{j=1}^{n} w_{j} \cdot E\left(\widetilde{S}_{W C}\left(\widetilde{\alpha}_{i j}\right)\right)_{\lambda}=\sum_{j=1}^{n} w_{j} \cdot\left[(1-\lambda) \cdot\left(\mu_{i j}^{-}-\nu_{i j}^{+}\right)+\lambda \cdot\left(\mu_{i j}^{+}-\nu_{i j}^{-}\right)\right]
$$

Example 3 (Continuation of Example 2). Assume the following BUM function $Q(y)=$ $y^{1 / 3}$, which results in the following attitudinal character value $\lambda=3 / 4$ and attitudinal expected score value matrix

$$
\left(E\left(\widetilde{S}_{W C}\left(\widetilde{\alpha}_{i j}\right)\right)_{3 / 4}\right)=\left(\begin{array}{cccc}
0.250 & 0.200 & 0.450 & 0.425 \\
0.500 & 0.550 & 0.300 & 0.300 \\
0.150 & 0.400 & 0.325 & 0.625 \\
0.300 & 0.300 & 0.600 & 0.450
\end{array}\right)
$$


The collective attitudinal expected score values are:

$$
s_{3 / 4}\left(A_{1}\right)=0.3275, s_{3 / 4}\left(A_{2}\right)=0.42, s_{3 / 4}\left(A_{3}\right)=0.3675, s_{3 / 4}\left(A_{4}\right)=0.510 .
$$

The final ranking of the alternatives would be:

$$
A_{4} \succ A_{2} \succ A_{3} \succ A_{1}
$$

The ranking of the alternatives depends on the value chosen for the attitudinal parameter $\lambda$, and therefore it would be interesting to know the conditions under which a change in the attitudinal value does not result in a reverse ordering of two alternatives. These conditions are given in the following sensitivity analysis theorem of the collective attitudinal expected score value (27).

Theorem 4. Let $\lambda$ be an attitudinal parameter value under which it has been established that $s_{\lambda}\left(A_{k}\right) \leq s_{\lambda}\left(A_{l}\right)$. Let $\Delta \lambda$ be a perturbation of the attitudinal character $\lambda$ with $0 \leq \lambda+\Delta \lambda \leq 1$. Then we have

$$
s_{\lambda+\Delta \lambda}\left(A_{k}\right) \leq s_{\lambda+\Delta \lambda}\left(A_{l}\right)
$$

iff

$$
\begin{cases}\max \left\{-\lambda, \frac{s_{\lambda}\left(A_{l}\right)-s_{\lambda}\left(A_{k}\right)}{\theta_{k}-\theta_{l}}\right\} \leq \Delta \lambda \leq 1-\lambda, & \text { if } \theta_{k}<\theta_{l} \\ -\lambda \leq \Delta \lambda \leq 1-\lambda, & \text { if } \theta_{k}=\theta_{l} \\ -\lambda \leq \Delta \lambda \leq \min \left\{1-\lambda, \frac{s_{\lambda}\left(A_{l}\right)-s_{\lambda}\left(A_{k}\right)}{\theta_{k}-\theta_{l}}\right\}, & \text { if } \theta_{k}>\theta_{l}\end{cases}
$$

where $\theta_{k}=\sum_{j=1}^{n} w_{j} \cdot\left(\mu_{k j}^{+}-\mu_{k j}^{-}+\nu_{k j}^{+}-\nu_{k j}^{-}\right)$and $\theta_{l}=\sum_{j=1}^{n} w_{j} \cdot\left(\mu_{l j}^{+}-\mu_{l j}^{-}+\nu_{l j}^{+}-\nu_{l j}^{-}\right)$.

Proof. Notice that $\Delta \lambda$ is subject to the following constraint:

$$
-\lambda \leq \Delta \lambda \leq 1-\lambda
$$

We have the following relation between $s_{\lambda+\Delta \lambda}\left(A_{k}\right)$ and $s_{\lambda}\left(A_{k}\right)$ :

$$
s_{\lambda+\Delta \lambda}\left(A_{k}\right)=s_{\lambda}\left(A_{k}\right)+\Delta \lambda \cdot \theta_{k}, \text { with } \theta_{k}=\sum_{j=1}^{n} w_{j} \cdot\left(\mu_{k j}^{+}-\mu_{k j}^{-}+\nu_{k j}^{+}-\nu_{k j}^{-}\right) .
$$

The following equivalence holds:

$$
s_{\lambda+\Delta \lambda}\left(A_{k}\right) \leq s_{\lambda+\Delta \lambda}\left(A_{l}\right) \Leftrightarrow \Delta \lambda \cdot\left(\theta_{k}-\theta_{l}\right) \leq s_{\lambda}\left(A_{l}\right)-s_{\lambda}\left(A_{k}\right) .
$$

Three scenarios are possible: 
- $\theta_{k}=\theta_{l}$. Because $s_{\lambda}\left(A_{k}\right) \leq s_{\lambda}\left(A_{l}\right)$ then (28) is true for any value of $\Delta \lambda$, i.e.

$$
-\lambda \leq \Delta \lambda \leq 1-\lambda
$$

- $\theta_{k}>\theta_{l} \Leftrightarrow \Delta \lambda \leq \frac{s_{\lambda}\left(A_{l}\right)-s_{\lambda}\left(A_{k}\right)}{\theta_{k}-\theta_{l}}$, and therefore:

$$
-\lambda \leq \Delta \lambda \leq \min \left\{1-\lambda, \frac{s_{\lambda}\left(A_{l}\right)-s_{\lambda}\left(A_{k}\right)}{\theta_{k}-\theta_{l}}\right\} .
$$

- $\theta_{k}<\theta_{l} \Leftrightarrow \Delta \lambda \geq \frac{s_{\lambda}\left(A_{l}\right)-s_{\lambda}\left(A_{k}\right)}{\theta_{k}-\theta_{l}}$, and therefore:

$$
\max \left\{-\lambda, \frac{s_{\lambda}\left(A_{l}\right)-s_{\lambda}\left(A_{k}\right)}{\theta_{k}-\theta_{l}}\right\} \leq \Delta \lambda \leq 1-\lambda .
$$

Example 4 (Continuation of Example 2). The general attitudinal expected score value matrix expression with respect to $\lambda$ is

$$
\left(E\left(\widetilde{S}_{W C}\left(\widetilde{\alpha}_{i j}\right)\right)_{\lambda}\right)=\left(\begin{array}{rrrr}
0.1+0.2 \cdot \lambda & -0.1+0.4 \cdot \lambda & 0.3+0.2 \cdot \lambda & 02+0.3 \cdot \lambda \\
0.2+0.4 \cdot \lambda & 0.4+0.2 \cdot \lambda & 0.4 \cdot \lambda & 0.4 \cdot \lambda \\
0.2 \cdot \lambda & 0.1+0.4 \cdot \lambda & 0.1+0.3 \cdot \lambda & 0.4+0.3 \cdot \lambda \\
0.4 \cdot \lambda & 0.4 \cdot \lambda & 0.3+0.3 \cdot \lambda & 0.3+0.2 \cdot \lambda
\end{array}\right)
$$

The collective attitudinal expected score values are:

$s_{\lambda}\left(A_{1}\right)=0.11+0.29 \cdot \lambda, s_{\lambda}\left(A_{2}\right)=0.18+0.32 \cdot \lambda, s_{\lambda}\left(A_{3}\right)=0.12+0.33 \cdot \lambda, s_{\lambda}\left(A_{4}\right)=0.15+0.38 \cdot \lambda$.

We observe that

$$
\begin{aligned}
& s_{\lambda}\left(A_{4}\right)>s_{\lambda}\left(A_{3}\right)>s_{\lambda}\left(A_{1}\right) \forall \lambda \Rightarrow A_{4} \succ A_{3} \succ A_{1} \forall \lambda \\
& s_{\lambda}\left(A_{2}\right)>s_{\lambda}\left(A_{3}\right)>s_{\lambda}\left(A_{1}\right) \forall \lambda \Rightarrow A_{2} \succ A_{3} \succ A_{1} \forall \lambda
\end{aligned}
$$

However, regarding the comparison of $A_{2}$ and $A_{4}$, we have the following

- If $\lambda \in[0,0.5)$ then $A_{2} \succ A_{4}$

- If $\lambda=0.5$ then $A_{2} \sim A_{4}$

- If $\lambda \in(0.5,1]$ then $A_{4} \succ A_{2}$ 
Summarising, we have the following:

- $\forall \lambda \in[0,0.5)$ we have: $A_{2} \succ A_{4} \succ A_{3} \succ A_{1}$.

- If $\lambda=0.5$ then $A_{2} \sim A_{4} \succ A_{3} \succ A_{1}$.

- $\forall \lambda \in(0.5,1]$ we have: $A_{4} \succ A_{2} \succ A_{3} \succ A_{1}$.

Therefore only when the value of $\lambda$ is close to 0.5 , we may have a reversal ordering of the alternatives $A_{2}$ and $A_{4}$. It is worth remarking here that the final ordering of the alternatives not only depends on the value of the attitudinal character but also on the particular criteria weighting vector, an issue that will be the focus of future research work.

The collective attitudinal expected score values results in a total ordering of the set of alternatives. For those alternatives with same collective attitudinal expected score values, we apply the second level ordering with the application of the collective attitudinal accuracy score value

$$
a_{\lambda}\left(A_{i}\right)=\sum_{j=1}^{n} w_{j} \cdot E\left(\widetilde{A}_{W C}\left(\widetilde{\alpha}_{i j}\right)\right)_{\lambda}=\sum_{j=1}^{n} w_{j} \cdot\left[(1-\lambda) \cdot\left(\mu_{i j}^{-}+\nu_{i j}^{-}\right)+\lambda \cdot\left(\mu_{i j}^{+}+\nu_{i j}^{+}\right)\right]
$$

An alternative expression to (29) for the collective attitudinal accuracy score value can be obtained if the relationship (20) between the the attitudinal expected accuracy score function and the attitudinal expected accuracy function is used:

$$
\begin{aligned}
a_{\lambda}\left(A_{i}\right) & =\sum_{j=1}^{n} w_{j} \cdot E\left(\widetilde{A}_{W C}\left(\widetilde{\alpha}_{i j}\right)\right)_{\lambda}=\sum_{j=1}^{n} w_{j} \cdot\left[E\left(\widetilde{S}_{W C}\left(\widetilde{\alpha}_{i j}\right)\right)_{\lambda}+\left(\nu_{i j}^{+}+\nu_{i j}^{-}\right)\right] \\
& =s_{\lambda}\left(A_{i}\right)+\sum_{j=1}^{n} w_{j} \cdot\left(\nu_{i j}^{+}+\nu_{i j}^{-}\right) .
\end{aligned}
$$

Example 5 (Continuation of Example 2). In Example 4, we had $s_{0.5}\left(A_{2}\right)=s_{0.5}\left(A_{2}\right)=$ 0.34. It is easy to prove that in this case we have that $a_{0.5}\left(A_{2}\right)=a_{0.5}\left(A_{2}\right)=0.74$, so we can not decide yet which alternative is the best. To do that, we apply the third level ordering based on the use of the membership uncertainty index. Obviously, a different criteria weighting vector to the one used in these examples might have led to different attitudinal expected accuracy values.

A similar reasoning to that of Theorem 4 can be used to prove the following sensitivity analysis of the collective attitudinal expected accuracy value (27): 
Theorem 5. Let $\lambda$ be an attitudinal parameter value under which it has been established that $a_{\lambda}\left(A_{k}\right) \leq a_{\lambda}\left(A_{l}\right)$. Let $\Delta \lambda$ be a perturbation of the attitudinal character $\lambda$ with $0 \leq \lambda+\Delta \lambda \leq 1$. Then we have

$$
a_{\lambda+\Delta \lambda}\left(A_{k}\right) \leq a_{\lambda+\Delta \lambda}\left(A_{l}\right)
$$

iff

$$
\begin{cases}\max \left\{-\lambda, \frac{a_{\lambda}\left(A_{l}\right)-a_{\lambda}\left(A_{k}\right)}{\theta_{k}-\theta_{l}}\right\} \leq \Delta \lambda \leq 1-\lambda, & \text { if } \theta_{k}<\theta_{l} \\ -\lambda \leq \Delta \lambda \leq 1-\lambda, & \text { if } \theta_{k}=\theta_{l} \\ -\lambda \leq \Delta \lambda \leq \min \left\{1-\lambda, \frac{a_{\lambda}\left(A_{l}\right)-a_{\lambda}\left(A_{k}\right)}{\theta_{k}-\theta_{l}}\right\}, & \text { if } \theta_{k}>\theta_{l}\end{cases}
$$

where $\theta_{k}=\sum_{j=1}^{n} w_{j} \cdot\left(\mu_{k j}^{+}-\mu_{k j}^{-}+\nu_{k j}^{+}-\nu_{k j}^{-}\right)$and $\theta_{l}=\sum_{j=1}^{n} w_{j} \cdot\left(\mu_{l j}^{+}-\mu_{l j}^{-}+\nu_{l j}^{+}-\nu_{l j}^{-}\right)$.

For those alternatives with same collective attitudinal expected score values and collective attitudinal accuracy score value, we apply the third level ordering with the computation of their respective collective membership uncertainty indexes.

$$
T\left(A_{i}\right)=\sum_{j=1}^{n} w_{j} \cdot\left[\left(\mu_{i j}^{+}-\mu_{i j}^{-}\right)-\left(\nu_{i j}^{+}-\nu_{i j}^{-}\right)\right] .
$$

Example 6 (Finishing Example 2). The collective membership uncertainty indexes corresponding to alternatives $A_{2}$ and $A_{4}$ are $T\left(A_{2}\right)=-0.04$ and $T\left(A_{4}\right)=0.04$, and therefore the final ranking of the set of alternatives when $\lambda=0.5$ would be:

$$
A_{2} \succ A_{4} \succ A_{3} \succ A_{1}
$$

\section{Conclusion}

Based on the concept of attitudinal character of a BUM and the continuous ordered weighted average (COWA) operator, attitudinal expected score and accuracy functions for IVIFNs are introduced and their relationship with existing score and accuracy degree functions are investigated. A set of properties are also proved, which makes them to be a type of weighted average operators. A key result provided is the construction of a total order on the set of IVIFNs using a three level ordering relation based on the conjunction application of the attitudinal expected score and accuracy functions and Wang et al.'s membership uncertainty index function. A resolution process of interval-valued intuitionistic fuzzy multi-attribute decision making problems that ranks the alternatives by taking 
accounting of the decision makers' risk attitude is also provided. It was remarked before that the final ordering of the alternatives not only depends on the value of the attitudinal character but also on the particular criteria weighting vector, which in conjunction with the development of consistency based decision models [47] will be the focus of future research work.

\section{Acknowledgements}

The authors are very grateful to the anonymous referees for their comments and suggestions. This work was supported by National Natural Science Foundation of China (NSFC) under the Grant No.71101131 and Zhejiang Provincial National Science Foundation for Distinguished Young Scholars of China under the Grant No. LR13G010001.

[1] K. Atanassov, Intuitionistic fuzzy sets, Fuzzy Sets and Systems 20 (1986), 87-96.

[2] L. A. Zadeh, Fuzzy sets, Information and Control 8 (1965), 338-353.

[3] J. Wu, F. Chiclana: A social network analysis trust-consensus based approach to group decision-making problems with interval-valued fuzzy reciprocal preference relations, Knowledge-Based Systems 59 (2014), 97-107.

[4] K. Atanassov and G. Gargov, Interval-valued intuitionistic fuzzy sets, Fuzzy Sets and Systems 31 (1989), 343-349.

[5] Z. J. Wang, K. W. Li and J. H. Xu, A mathematical programming approach to multi-attribute decision making with interval-valued intuitionistic fuzzy assessment information, Expert Systems with Applications 38 (2011), 12462-12469.

[6] Y. J. Yang and F. Chiclana, Intuitionistic fuzzy sets: Spherical representation and distances, International Journal of Intelligent Systems 24 (2009), 399-420.

[7] Y. J. Yang and F. Chiclana,, Consistency of 2D and 3D distances of intuitionistic fuzzy sets, Expert Systems with Applications 39 (2012), 8665-8670.

[8] J. Wu and F. Chiclana, Non-dominance prioritization methods for intuitionistic and interval-valued intuitionistic preference relations, Expert Systems with Applications 39 (2012), 13409-13416 
[9] J. Wu, H.B.Huang and Q.W.Cao, Research on AHP with interval-valued intuitionistic fuzzy sets and its application in multi-criteria decision making problems, Applied Mathematical Modelling 37 (2013), 9898-9906

[10] J. Wu and Y.J. Liu, An approach for multiple attribute group decision making problems with interval-valued intuitionistic trapezoidal fuzzy numbers, Computers and Industrial Engineering 66(2013), 311-324

[11] T. Y. Chen, Multiple criteria group decision-making with generalized interval-valued fuzzy numbers based on signed distances and incomplete weights, Applied Mathematical Modelling 36 (2012), 3029-3052.

[12] Z. L. Yue, An approach to aggregating interval numbers into interval-valued intuitionistic fuzzy information for group decision making, Expert Systems with Applications 38 (2011), 6333-6338.

[13] F. Ye, An extended TOPSIS method with interval-valued intuitionistic fuzzy numbers for virtual enterprise partner selection, Expert Systems with Applications 37 (2010), 7050-7055.

[14] J. Ashayeri, G. Tuzkaya and U. R. Tuzkaya, Supply chain partners and configuration selection: An intuitionistic fuzzy Choquet integral operator based approach, Expert Systems with Applications 39 (2012), 3642-3649.

[15] K.Devi, Extension of VIKOR method in intuitionistic fuzzy environment for robot selection, Expert Systems with Applications 38 (2011), 14163-14168.

[16] R. Saadati, S. M. Vaezpour and Y. J. Choc, Quicksort algorithm: Application of a fixed point theorem in intuitionistic fuzzy quasi-metric spaces at a domain of words, Journal of Computational and Applied Mathematics 228 (2009), 219-225.

[17] F. Chiclana, E. Herrera-Viedma, F. Herrera, S. Alonso: Some induced ordered weighted averaging operators and their use for solving group decision-making problems based on fuzzy preference relations, European Journal of Operational Research 182 (1) (2007), 383-399.

[18] Z. S. Xu, Intuitionistic fuzzy aggregation operators, IEEE Transactions on Fuzzy Systems 15 (2007), 1179-1187. 
[19] D. F. Li, The GOWA operator based approach to multiattribute decision making using intuitionistic fuzzy sets, Mathematical and Computer Modelling 53 (2011), 1182-1196.

[20] Z. S. Xu, Methods for aggregating interval-valued intuitionistic fuzzy information and their application to decision making, Control and Decision 22 (2007), 215-219.

[21] J. Wu and Q. W. Cao, Same families of geometric aggregation operators with intuitionistic trapezoidal fuzzy numbers, Applied Mathematical Modelling 37 (2013), $318-327$.

[22] C. Q. Tan and X. H. Chen, Intuitionistic fuzzy Choquet integral operator for multicriteria decision making, Expert Systems with Applications 37 (2010), 149-157.

[23] C. Q. Tan, A multi-criteria interval-valued intuitionistic fuzzy group decision making with Choquet integral-based TOPSIS, Expert Systems with Applications 38 (2011), 3023-3033.

[24] T. Y. Chen, A comparative analysis of score functions for multiple criteria decision making in intuitionistic fuzzy settings, Information Sciences 181 (2011), 3652-3676.

[25] S. M. Chen and J. M. Tan, Handling multi-criteria fuzzy decision-making problems based on vague set theory, Fuzzy Sets and Systems 67 (1994), 163-172.

[26] D. H. Hong and C.H. Choi, Multicriteria fuzzy decision-making problems based on vague set theory, Fuzzy Sets and Systems 114 (2000), 103-113.

[27] J. Q. Wang, L. Y. Meng and X. H. Cheng, Multi-criteria decision making method based on vague sets and risk attitudes of decision makers, Systems Engineering and Electronics 2 (2009), 361-365.

[28] K. Atanassov, G. Pasi and R. R. Yager, Intuitionistic fuzzy interpretations of multicriteria multi-person and multi-measurement tool decision making, International Journal of Systems Science 36 (2005), 859-868.

[29] T. Y. Chen, An outcome-oriented approach to multicriteria decision analysis with intuitionistic fuzzy optimistic/pessimistic operators, Expert Systems with Applications 37 (2010), 7762-7774. 
[30] S. P. Wan, and J. Y. Dong, A possibility degree method for interval-valued intuitionistic fuzzy multi-attribute group decision making, Journal of Computer and System Sciences 80 (2014), 237-256.

[31] Z. J. Wang, K. W. Li and W. Wang, An approach to multiattribute decision making with interval-valued intuitionistic fuzzy assessments and incomplete weights, Information Sciences 179 (2009), 3026-3040.

[32] J. Q. Wang, K. J. Li and H. Y. Zhang, Interval-valued intuitionistic fuzzy multicriteria decision-making approach based on prospect score function, KnowledgeBased Systems 27 (2012), 119-215.

[33] S. P. Wan, Q. Y. Wang, and J. Y. Dong, The extended VIKOR method for multiattribute group decision making with triangular intuitionistic fuzzy numbers, Knowledge Based Systems 52 (2013), 65-77.

[34] S. P. Wan, Multi-attribute decision making method based on possibility variance coefficient of triangular intuitionistic fuzzy numbers, International Journal of Uncertainty, Fuzziness and Knowledge-Based Systems 21 (2014), 223-243.

[35] P.D. Liu, and J. Y. Dong, Methods for Aggregating Intuitionistic Uncertain Linguistic variables and Their Application to Group Decision Making, Information Sciences 205(2012), 58-71.

[36] P.D. Liu, Some Generalized Dependent Aggregation Operators with Intuitionistic Linguistic Numbers and Their Application to Group Decision Making, Journal of Computer and System Sciences 79(2013), 131-143.

[37] P.D. Liu, and Y. M. Wang, Multiple Attribute Group Decision Making Methods Based on Intuitionistic Linguistic Power Generalized Aggregation Operators, Applied soft computing 17(2014), 90-104.

[38] Z. S. Xu and J. Chen, An approach to group decision making based on interval-valued intuitionistic judgment matrices, System Engineer-Theory $\&$ Practice 27 (2007), 126133. 
[39] J. Ye, Multicriteria fuzzy decision-making method based on a novel accuracy function under interval-valued intuitionistic fuzzy environment, Expert Systems with Applications 36 (2009), 6899-6902.

[40] V. Lakshmana Gomathi Nayagama and G. Sivaraman, Ranking of interval-valued intuitionistic fuzzy sets, Applied Soft Computing 11 (2011), 3368-3372.

[41] R. R. Yager, OWA aggregation over a continuous interval argument with applications to decision making, IEEE Transactions on Systems, Man, and Cybernetics-Part B: Cybernetics 34 (2004), 1952-1963.

[42] M. Hanss, Applied Fuzzy Arithmetic. An Introduction with Engineering Applications. Springer-Verlag Berlin Heidelberg, 2005.

[43] P. Pérez-Asurmendi, F. Chiclana. Linguistic majorities with difference in support. Applied Soft Computing, 18 (2014) 196-208.

[44] L.A. Zadeh. Outline of a new approach to the analysis of complex systems and decision processes, IEEE Transactions on Systems, Man and Cybernetics, 3, 28-44, 1973.

[45] M. Brunelli, J. Mezei. How different are ranking methods for fuzzy numbers? A numerical study, International Journal of Approximate Reasoning, 54 (5), 627-639, 2013.

[46] R. Yager. Quantifier guided aggregation using owa operators. International Journal of Intelligent Systems 11(1), 1996, 49-73.

[47] F. Chiclana, E. Herrera-Viedma, S. Alonso, F. Herrera: Cardinal consistency of reciprocal preference relations: a characterization of multiplicative transitivity. IEEE Transactions on Fuzzy Systems 17 (1) (2009), 14-23. 